\section{Revista de CIENCIAS AMBIENTALES Tropical Journal of Environmental Sciences}

Revista de Ciencias Ambientales (Trop J Environ Sci) e-ISSN: $2215-3896$

(Julio-Diciembre, 2021) . Vol 55(2): 197-228

DOI: https://doi.org/10.15359/rca.55-2.10

Open Access: www.revistas.una.ac.cr/ambientales e-mail: revista.ambientales@una.ac.cr Zuluaga-Carrero J. y Renjifo L.

\title{
Cambios en la composición de aves en diferentes fisionomías de vegetación en un enclave seco en Colombia
}

\author{
Bird composition changes in different vegetation physiognomies \\ of a dry enclave in Colombia
}

Juliana Zuluaga-Carrero' ${ }^{1}$ Luis Miguel Renjifo ${ }^{2}$

[Recibido: 16 de mayo 2020, Aceptado: 27 de abril 2021, Corregido: 26 de mayo 2021, Publicado: 1 de julio 2021]

\section{Resumen}

[Introducción]: La marcada estacionalidad del enclave seco favorece endemismos y especies de distribución restringida, aunque durante el periodo de lluvias esta distribución puede variar, siendo más amplia para aprovechar recursos de otros ecosistemas. [Objetivo]: El presente trabajo tiene el propósito de estudiar las variaciones que ocurren en la avifauna diferentes fisionomías en un gradiente altitudinal en un bosque seco durante las lluvias. [Metodología]: Se utilizaron 72 unidades muestrales simultáneas para aves y plantas en nueve sitios pertenecientes a tres fisionomías de vegetación (bosques riparios, de ladera y matorrales) en tres franjas de altitud, establecimos dos variables dependientes, abundancia y riqueza; y cuatro variables independientes: riqueza de árboles, área basal, franja de altitud y sitio. [Resultados]: Se encontró que la franja alta corresponde con el bioma azonal del zonobioma húmedo y tiene mayor riqueza de avifauna y menor riqueza florística, mientras que la zona baja representa un zonobioma alternohídrico tropical, con menor diversidad de avifauna y alta diversidad florística. La menor riqueza de aves se encuentra en los bosques riparios y la mayor en los bosques de ladera, con un bajo recambio en esta última fisionomía. [Conclusiones]: Durante el periodo de lluvias hay un bajo recambio altitudinal de especies en los bosques de ladera y esto puede explicarse por la heterogeneidad florística, así mismo, los bosques riparios merecen atención por su baja riqueza de aves y plantas, aspectos que podrían considerarse en el establecimiento de áreas protegidas en ecosistemas secos.

Palabras clave: Avifauna; bosque seco tropical; conservación; gradiente altitudinal; valle del río Chicamocha.

\section{Abstract}

[Introduction]: Distinct seasonality of the dry enclave benefit endemisms and species with restricted distribution, although during rainy season this distribution may vary, being broader to take advantage of resources from other ecosystems. [Objective]: Our aim study has studying variations occurring in birds in different physiognomies existing in an elevation gradient in a tropical dry forest during rain period. [Methodology]: We used 72 simultaneous sampling units for birds and plants in nine sites belonging to three vegetation physiognomies (riparian, hillside and scrub forests) in three altitude bands, we established two response variables, bird abundance and richness, and four independent variables: tree richness, basal area, altitude band and site. [Results]: We found that the upper band corresponds to the azonal orobiome of the tropical humid zonobiome and has greater richness of avifauna and less floristic richness, while the lower zone represents a tropical alternohydric zonobiome, with less diversity of birds

1 Departamento de Ecología y Territorio, Facultad de Estudios Ambientales y Rurales, Pontificia Universidad Javeriana, Bogotá, Colombia; zuluaga.juliana@javeriana.edu.co, http://orcid.org/0000-0002-9208-5958

2 Departamento de Ecología y Territorio, Facultad de Estudios Ambientales y Rurales, Pontificia Universidad Javeriana, Bogotá, Colombia; lmrenjifo@javeriana.edu.co, http://orcid.org/0000-0003-1907-2760

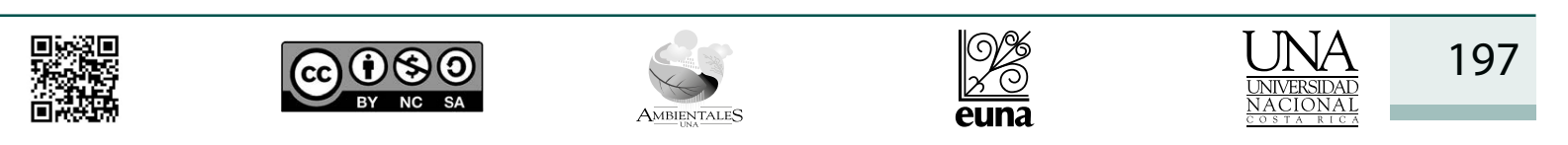




\section{Revista de CIENCIAS AMBIENTALES Tropical Journal of Environmental Sciences}

Revista de Ciencias Ambientales (Trop J Environ Sci)

e-ISSN: 2215-3896

(Julio-Diciembre, 2021) . Vol 55(2): 197-228

DOI: https://doi.org/10.15359/rca.55-2.10

Open Access: www.revistas.una.ac.cr/ambientales e-mail: revista.ambientales@una.ac.cr Zuluaga-Carrero J. y Renjifo L.

and high floristic diversity. The least richness of birds was found in riparian forests, and the greatest in hillside forests, with a low turnover. [Conclusions]: During rainy season there is a low altitudinal turnover of species in the hillside forests and maybe can be explained by floristic heterogeneity, likewise, riparian forests deserve attention due to their low bird richness and plants, aspects that could be considered in the establishment of protected areas in dry ecosystems.

Keywords: Avifauna, tropical dry forest, conservation, altitudinal gradient; Chicamocha valley river.

\section{Introducción}

El bosque seco estacional neotropical (bosque seco tropical) ha sido drásticamente transformado como consecuencia de las actividades antrópicas, a pesar de esto, aún yacen remanentes con una diversidad florística exclusiva a nivel regional (Banda- $\mathrm{R}$ et al., 2016), un aspecto de alta importancia para la conservación de sus especies de aves. Algunos estudios señalan que la complejidad estructural de la vegetación incrementa la heterogeneidad del hábitat y, consecuentemente, aumenta la diversidad de aves (Wang et al., 2014). Entender la relación entre las aves y su hábitat es fundamental para plantear estrategias de conservación que permitan evitar la pérdida de especies a causa de la fragmentación acelerada que sufren hoy los bosques (Bregman et al., 2014). La selección del hábitat por parte de las aves no depende únicamente de un factor, sino que está determinada por una combinación de ellos entre los que se encuentran: cobertura, diversidad, composición florística y estructura de la vegetación; esta última, reconocida como un factor notable para la composición y un indicador para la selección de hábitat en aves (Müller et al., 2010).

Los árboles con mayores diámetros en sus troncos usualmente están asociados con bosques que cuentan con una compleja estructura, conformada por epífitas, plantas de sotobosque y numerosos vertebrados (Vázquez \& Renton, 2015). Algunos estudios han usado el diámetro de los árboles como una variable que explica su estructura, por su relación con especies de aves que forrajean y nidifican en cavidades y que forrajean en el suelo (Melo et al., 2020), siendo, por tanto, una variable a considerar. Al comparar estos bosques con aquellos que tienen árboles con menores diámetros, las aves pueden encontrar una mayor disponibilidad de alimento, sitios de protección contra las variaciones ambientales y depredadores, así como también, una mayor disponibilidad de recursos para construir nidos, hecho que favorece su riqueza (Santamaría-Rivero et al., 2016).

En los bosques secos, la estacionalidad durante las lluvias causa cambios en la vegetación, particularmente en especies caducifolias, lianas y en la cobertura de herbáceas (Avalos \& Mulkey, 1999). En este tiempo existe una mayor producción de biomasa, mayor cantidad de recursos y algunas aves, inclusive, sincronizan la temporada con su reproducción; este incremento en la oferta de alimento permite alimentar a los polluelos, como ocurre por ejemplo en Coereba flaveola, por lo que durante esta época además es posible observar un aumento en la abundancia de algunas especies (Brown \& Sherry, 2005). Por el contrario, a lo largo de la época seca, hay

\begin{tabular}{|c|c|c|}
\hline 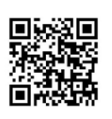 & (c) (1) & 198 \\
\hline
\end{tabular}




\section{Revista de CIENCIAS AMBIENTALES Tropical Journal of Environmental Sciences}

Revista de Ciencias Ambientales (Trop J Environ Sci)

e-ISSN: 2215-3896

(Julio-Diciembre, 2021) . Vol 55(2): 197-228

DOI: https://doi.org/10.15359/rca.55-2.10

Open Access: www.revistas.una.ac.cr/ambientales e-mail: revista.ambientales@una.ac.cr Zuluaga-Carrero J. y Renjifo L.

escasez de agua, los árboles caducifolios pierden sus hojas y hay una menor disponibilidad de recursos para las aves (Murphy \& Lugo, 1987). En la temporada seca algunas especies de aves deben moverse hacia otros tipos de vegetación en los cuales puedan encontrar agua, alimento y refugio (Vázquez et al., 2009). Siendo, por ende, indispensable conocer los cambios que ocurren en la composición de aves durante la época de lluvias en un área que ha sido poco estudiada, como son los bosques secos del Cañón del Chicamocha, Colombia, un sitio que demás requiere medidas prioritarias de conservación (Collazos-González et al., 2020).

En los bosques secos las especies de aves no están presentes solamente en las franjas remanentes o en los pequeños parches existentes, pues su presencia podría estar dependiendo de ecosistemas cercanos en los que se encuentran recursos complementarios como los ecosistemas riparios y montanos (Anderson et al., 2011). Se ha observado que durante la época lluviosa las aves pueden tener una distribución más amplia en comparación con la época seca, en la cual están limitadas principalmente a los bosques riparios en donde hay una mayor disponibilidad de agua (McKinnon et al., 2015). Entonces, estudiar las comunidades de aves presentes en diferentes tipos de vegetación durante la época de lluvias puede brindar información valiosa para lograr comprender variaciones en la composición de especies y, a su vez, servir de referencia para futuros estudios que se realicen durante la época seca.

En este estudio se evaluó la diversidad composicional de las comunidades de aves en nueve tipos de vegetación en el enclave seco del cañón del río Chicamocha en Santander, Colombia. Nuestro objetivo fue explorar las diferencias que se presentan con los cambios de altitud y las fisionomías de vegetación. Nuestra hipótesis señala que hay cambios en la composición de aves entre tipos de vegetación con la altitud, los cuales pueden estar relacionados con la estructura de la vegetación y la composición florística (Sitters et al., 2016). En la medida en que logremos comprender cuáles factores pueden estar determinando la selección del hábitat en las aves, se podrán lograr direccionar esfuerzos hacia su conservación. Con este estudio queremos destacar la necesidad de incluir diferentes tipos de vegetación en el planteamiento de estrategias de conservación para las aves del bosque seco tropical.

\section{Metodología}

\subsection{Descripción del área de estudio}

El área de estudio hace parte del enclave seco del Cañón del río Chicamocha, en el noroccidente de la cordillera Oriental de los Andes Colombianos, departamento de Santander, municipio de los Santos, cerca al sitio en donde el río Chicamocha se vuelve el Sogamoso, $6^{\circ} 88^{\prime} \mathrm{N} \mathrm{y} 73^{\circ} 17^{\prime} \mathrm{W}$. El área tiene un régimen bimodal de precipitación con un promedio anual de $730.8 \mathrm{~mm}$. Esta zona se encuentra en un rango altitudinal entre los 300 y $1600 \mathrm{~m}$, y su temperatura puede oscilar entre 21 y 40 ${ }^{\circ} \mathrm{C}$ en un día (observaciones personales), con un promedio anual de $24.4{ }^{\circ} \mathrm{C}$ (Albesiano \& Fernández-Alonso, 2006).

\begin{tabular}{|c|c|c|c|c|c|}
\hline 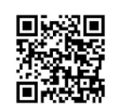 & (c) () () () & 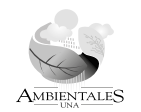 & $\frac{1 \%}{2 \%}$ & 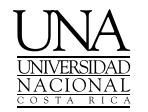 & 199 \\
\hline
\end{tabular}




\section{Revista de CIENCIAS AMBIENTALES Tropical Journal of Environmental Sciences}

Revista de Ciencias Ambientales (Trop J Environ Sci) e-ISSN: 2215-3896

(Julio-Diciembre, 2021) . Vol 55(2): 197-228 DOI: https://doi.org/10.15359/rca.55-2.10 Open Access: www.revistas.una.ac.cr/ambientales e-mail: revista.ambientales@una.ac.cr Zuluaga-Carrero J. y Renjifo L.

El enclave seco del cañón del río Chicamocha cuenta con 15.000 hectáreas en un gradiente altitudinal entre 300-1580 m s.n.m. Hay dos biomas: el primero, el zonobioma alternohígrico tropical, en la franja de altitud baja y media entre los 300-920 m s.n.m., caracterizado por especies de árboles como Haematoxylum brasiletto, Astronium graveolens, Eugenia biflora y Bursera simaruba. Los suelos de este bioma tienen mayormente areniscas, con erosión fuerte en los que se han llevado a cabo actividades de pastoreo caprino, con áreas que han estado abandonadas entre 1-3 años. El segundo, el orobioma azonal del zonobioma húmedo tropical, en la franja de altitud alta entre los 1150-1580 m s.n.m., caracterizado por especies de árboles como Calliandra purdiei, Ficus insipida, Myrsine guianensis y Vismia baccifera. Sus suelos varían entre limolitas y calcitas, con erosión severa a moderada, en los que se llevan a cabo actividades de pastoreo vacuno, caprino y ovino, y en algunas áreas hay cultivos de tabaco (Nicotiana tabacum) y maracuyá (Passiflora edulis), así como extracción de madera. En la franja media y baja también hay escarpes rocosos con suelo descubierto y pendientes abruptas en las que ocurren deslizamientos de suelo por erosión. Ambos biomas considerados para este estudio como bosque seco tropical ya que la vegetación encontrada queda cobijada bajo esta definición por su marcada estacionalidad en el periodo de las lluvias y sus ecosistemas xerofíticos y subxerofíticos (Rodríguez et al., 2006).

\subsection{Selección de los sitios de muestreo}

La selección se hizo a partir de imágenes de satélite y visitas previas de campo. Dentro de los criterios de selección se incluyó: altitud y fisionomía dominante (bosque ripario, bosque de ladera y matorral), presencia o no de cuerpos de agua, pendiente, erosión, especies dominantes de árboles y uso del suelo. A partir de estos criterios fueron seleccionados en total nueve sitios (9) en cada uno con ocho unidades de muestreo (Figuras 1 y 2). En total se establecieron 72 unidades de muestreo, 24 por franja altitudinal, con tres repeticiones cada una en el caso de las aves, lo que tomó tres meses consecutivos de muestreo. La fase de campo se hizo entre septiembre y noviembre del 2014, coincidiendo con la época de lluvias en la región.

\begin{tabular}{|c|c|c|c|c|c|}
\hline 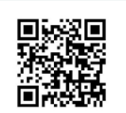 & (c) (i) $(0)$ & $\underset{\text { AMBIINTALS }}{\infty}$ & $\frac{\mid \% \%}{\text { euna }}$ & $\frac{\text { UNA }}{\frac{\text { UNILERIDAD }}{\text { NACIONAL }}}$ & 200 \\
\hline
\end{tabular}




\section{Revista de CIENCIAS AMBIENTALES Tropical Journal of Environmental Sciences}

Revista de Ciencias Ambientales (Trop J Environ Sci) e-ISSN: 2215-3896

(Julio-Diciembre, 2021) . Vol 55(2): 197-228 DOI: https://doi.org/10.15359/rca.55-2.10 Open Access: www.revistas.una.ac.cr/ambientales e-mail: revista.ambientales@una.ac.cr Zuluaga-Carrero J. y Renjifo L.
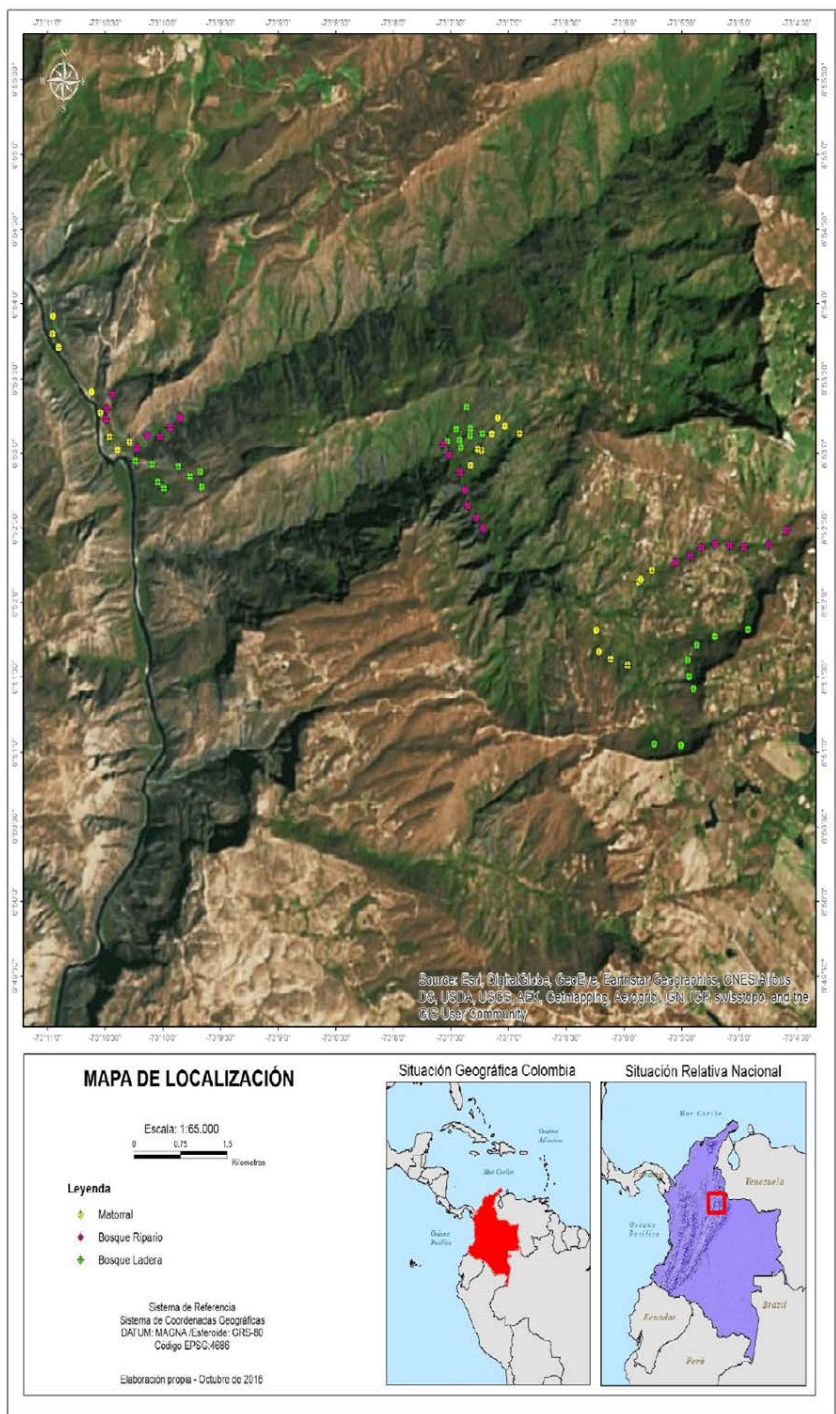

Figura 1. Mapa del área de estudio en el enclave seco del cañón del río Chicamocha, Colombia, se muestra la ubicación geográfica del sitio y 72 unidades de muestreo. Se establecieron ocho (8) puntos en cada una de las tres (3) fisionomías de vegetación, los puntos rosa corresponden a bosques riparios, verde bosques de ladera y amarillos a vegetación de matorral, para un total de nueve sitios distintos.

Figure 1. Map shows the study area in the dry enclave of the Chicamocha river canyon, Colombia with geographic location of the site and 72 sampling units. Eight (8) points were established in each of the three (3) vegetation physiognomies, the pink points correspond to riparian forests, green hillside forests and yellow to scrub vegetation, for a total of nine different sites.

\begin{tabular}{|c|c|c|}
\hline 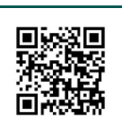 & (c) (i) () () () & 201 \\
\hline
\end{tabular}




\section{Revista de CIENCIAS AMBIENTALES Tropical Journal of Environmental Sciences}

Revista de Ciencias Ambientales (Trop J Environ Sci)

e-ISSN: 2215-3896

(Julio-Diciembre, 2021). Vol 55(2): 197-228

DOI: https://doi.org/10.15359/rca.55-2.10

Open Access: www.revistas.una.ac.cr/ambientales e-mail: revista.ambientales@una.ac.cr Zuluaga-Carrero J. y Renjifo L.

Los bosques riparios son franjas entre 10 y $30 \mathrm{~m}$ de ancho, con árboles de $20 \mathrm{~m}$ de altura promedio que se establecen bordeando una quebrada, los cuales varían de nombre dependiendo de su altitud. En la zona alta los sitios se establecieron en la quebrada La Purnia, en la zona media en la quebrada Honda y en la zona baja en la quebrada El Duende. La pendiente de este tipo de vegetación puede variar entre moderadamente quebrada (25-50\%) y moderadamente escarpada (50-75 \%), con alta cantidad de hojarasca y rocas. El flujo de agua es permanente durante la época de lluvias e intermitente en el periodo seco.
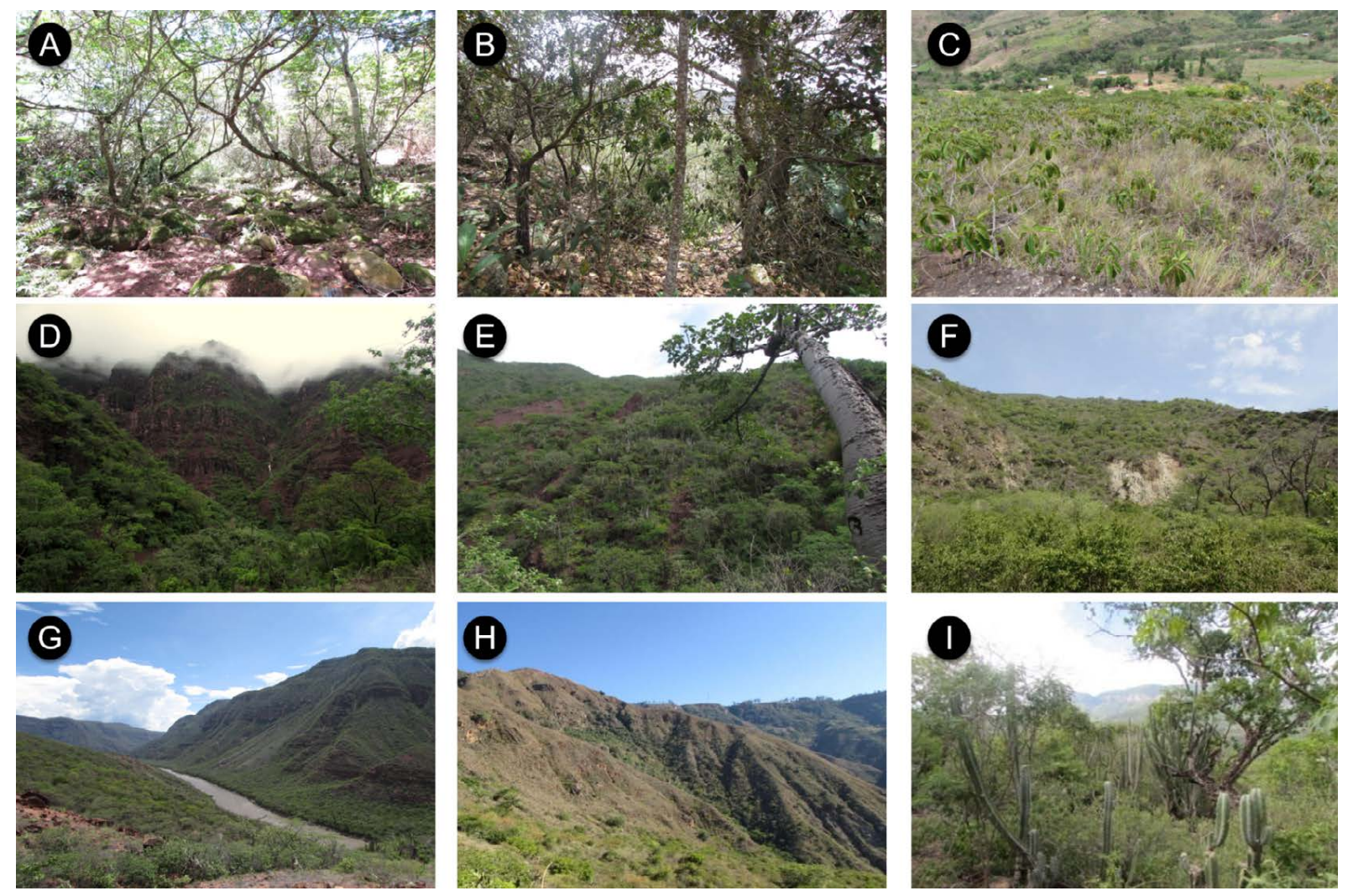

Figura 2. Sitios muestreadas en el enclave seco del cañón del río Chicamocha, Colombia. Se muestrearon nueve tipos de vegetación correspondientes a tres franjas de altitud, la zona alta entre 1150-1580 m s.n.m. (A-C), la zona media entre 641-1021 m s.n.m. (D-F) y la zona baja entre 317-625 m s.n.m. (G-I), cada franja con tres fisionomías de vegetación distintas, bosques riparios (A, D y G), bosques de ladera (B, E y H) y matorrales (C, F e I).

Figure 2. Sites sampled in the dry enclave of the Chicamocha river canyon, Colombia. Nine types of vegetation corresponding to three altitude bands were sampled, the upper zone between 1150-1580 $\mathrm{m}$ a.s.l. (A-C), the middle zone between 641-1021 m a.s.l. (D-F) and the low zone between 317-625 m a.s.l. (G-I), each strip with three different vegetation physiognomies, riparian forests $(A, D$ and $G)$, hillside forests $(B, E$ and $H)$ and scrub $(C, F$ and I).

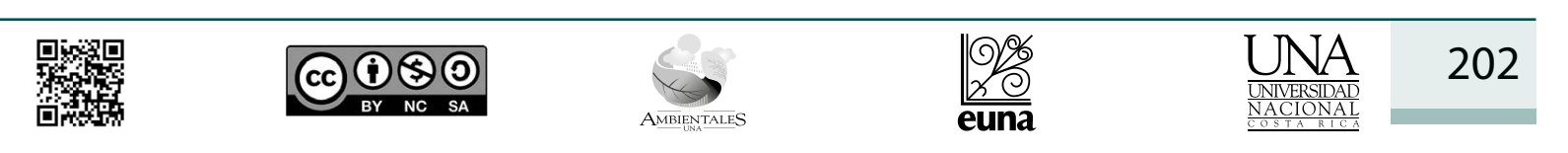




\section{Revista de CIENCIAS AMBIENTALES Tropical Journal of Environmental Sciences}

Revista de Ciencias Ambientales (Trop J Environ Sci)

e-ISSN: 2215-3896

(Julio-Diciembre, 2021) . Vol 55(2): 197-228

DOI: https://doi.org/10.15359/rca.55-2.10

Open Access: www.revistas.una.ac.cr/ambientales e-mail: revista.ambientales@una.ac.cr Zuluaga-Carrero J. y Renjifo L.

Los bosques de ladera se establecen en los escarpes pendientes donde se encuentran franjas continuas con 100-200 hectáreas de bosque con árboles de $15 \mathrm{~m}$ de altura promedio. La pendiente de este tipo de vegetación es fuertemente escarpada ( $>75 \%)$, en estos bosques no hay cuerpos de agua, aunque durante las lluvias torrenciales se forman cuerpos de agua temporales. El matorral por su parte tiene franjas entre 50-80 hectáreas de vegetación secundaria, se establece como consecuencia del abandono de tierras productivas en la zona alta y media, aunque en la zona baja es un tipo de vegetación natural; son áreas poco extensas que tienen vegetación con una altura promedio de siete metros.

\subsection{Métodos para vegetación}

Para estudiar las características de cada sitio se establecieron transectos de $50 \times 2 \mathrm{~m}$. En cada uno se midieron todos los árboles con diámetro a la altura del pecho - DAP $\geq 10 \mathrm{~cm}$, se establecieron ocho transectos por cada tipo de vegetación con el propósito de conocer el área basal por hectárea de cada uno; dicha variable fue seleccionada por su relación con la riqueza de aves (Melo et al., 2020; Santamaría-Rivero et al., 2016). Para la identificación de las especies de árboles se colectaron y procesaron dos muestras por cada especie no conocida y, posteriormente, estas fueron identificadas con listados de referencia (Albesiano \& Fernández-Alonso, 2006). Se usaron fotografías del herbario virtual de bosques secos de Colombia y el portal web del Herbario Nacional Colombiano, asimismo, se contó con la colaboración de expertos botánicos que han trabajado en la zona de estudio.

\subsection{Métodos para aves}

Para caracterizar la avifauna, se utilizó la metodología de puntos de conteo, tomando el centro de cada transecto de vegetación, para lograr unidades simultáneas de aves y vegetación. Cada punto de conteo con un radio fijo de $25 \mathrm{~m}$ con una distancia entre puntos de $200 \mathrm{~m}$ de separación. En cada punto, se hicieron tres conteos en días distintos, cada punto con una duración de 10 minutos, se registraron observaciones y vocalizaciones, se detectaron los individuos por especie dentro del radio. Estos registros fueron considerados como la media de las detecciones hechas en cada punto (Ralph et al., 1996). Las observaciones se realizaron entre las 06h00-09h30 y entre las 15h-17h30, para un total de siete horas de esfuerzo para cada punto. Durante este tiempo se registró solo aquellas aves que estuvieran usando la vegetación, sin incluir aquellas que estuvieran por fuera de los puntos o sobrevolando. Se asignó la categoría de migración y endemismo según Chaparro-Herrera et al. (2013) y la categoría de conservación, según la clasificación propuesta por IUCN (Renjifo et al., 2017).

\subsection{Análisis de datos}

Para cumplir con los dos objetivos propuestos se plantearon dos variables dependientes, riqueza y abundancia de aves, así como cuatro posibles variables independientes: riqueza de

\begin{tabular}{|c|c|c|}
\hline 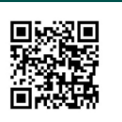 & (c) (i) (2) () & 203 \\
\hline
\end{tabular}




\section{Revista de CIENCIAS AMBIENTALES Tropical Journal of Environmental Sciences}

Revista de Ciencias Ambientales (Trop J Environ Sci)

e-ISSN: 2215-3896

(Julio-Diciembre, 2021). Vol 55(2): 197-228

DOI: https://doi.org/10.15359/rca.55-2.10

Open Access: www.revistas.una.ac.cr/ambientales e-mail: revista.ambientales@una.ac.cr Zuluaga-Carrero J. y Renjifo L.

árboles, área basal, franja de altitud y sitio. A partir de estas variables se evaluó, primeramente, si la estructura en términos del área basal de la vegetación está teniendo influencia en las aves, o si, por el contrario, es la diversidad florística la que contribuye a esta variación. Con el fin de calcular el total de los muestreos de plantas y aves, se hicieron análisis de rarefacción y se calcularon estimadores no paramétricos ACE y CHAO 1 para calcular la representatividad de los muestreos (Chazdon et al., 1998), lo anterior usando RStudio.

\subsubsection{Comunidades de plantas}

Para conocer la forma en la que varían las comunidades de plantas entre los nueve sitios, se analizaron las diferencias en cuanto a la riqueza y abundancia de plantas. Para evaluar la estructura se tomó en cuenta el DAP, con el cual se calculó el área basal por sitio usando la siguiente ecuación:

$$
\pi^{*} \mathrm{DAP}^{2} / 4(\mathbf{E . 1})
$$

Donde:

$\pi:$ pi, 3.14

DAP: Diámetro altura de pecho, medido a $1.3 \mathrm{~m}$

A esta variable se le hizo la prueba no paramétrica Friedman para conocer las diferencias y se calculó el índice de similitud de Bray-Curtis para observar cambios en la composición de plantas entre sitios.

\subsubsection{Comunidades de aves}

Las variaciones en el número de individuos y especies de aves se analizaron mediante diferentes pruebas. Se usó la prueba paramétrica ANDEVA para observar variaciones en la riqueza de las aves; se usó la prueba no paramétrica Friedman con las especies con más de 10 registros para asignarlas a una fisionomía de vegetación general (bosques y/o matorrales). También, se construyeron curvas rango abundancia (Izsák \& Pavoine, 2012), para evaluar cuatro modelos rango-abundancia, vara partida, normal logarítmico, serie logarítmico y serie geométrico (Gotelli \& Graves, 1996). Se calculó el índice de similitud de Bray-Curtis y la tasa de recambio de especies con índice de diversidad beta $\beta-3$, lo anterior para considerar su desempeño en gradientes altitudinales (Koleff et al., 2003), tanto los análisis como los diagramas se realizaron con el programa PAST.

\subsubsection{Comunidades de aves y tipos de vegetación}

Para evaluar la posible relación entre plantas y aves en cada sitio se realizó un análisis de escalamiento multidimensional, el cual muestra los datos desde el espacio dimensional que estos ocupan, buscando la mejor manera en la cual se organiza su estructura en relación con

\begin{tabular}{|c|c|c|c|c|c|}
\hline 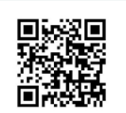 & (c) (i) $(0)$ & $\underset{\text { AMBIINTALS }}{\infty}$ & $\frac{\mid \% \%}{\text { euna }}$ & $\frac{\text { UNA }}{\frac{\text { UNILERIDAD }}{\text { NACIONAL }}}$ & 204 \\
\hline
\end{tabular}




\section{Revista de CIENCIAS AMBIENTALES Tropical Journal of Environmental Sciences}

Revista de Ciencias Ambientales (Trop J Environ Sci)

e-ISSN: 2215-3896

(Julio-Diciembre, 2021). Vol 55(2): 197-228

DOI: https://doi.org/10.15359/rca.55-2.10

Open Access: www.revistas.una.ac.cr/ambientales e-mail: revista.ambientales@una.ac.cr Zuluaga-Carrero J. y Renjifo L.

las variables propuestas. Para evaluar la relación entre las variables de las aves y las plantas se estimaron modelos lineares generalizados. En estos modelos, se evaluaron dos variables independientes: riqueza y abundancia de aves. Así, también, se tomó en consideración la relación de estas con las siguientes variables dependientes: sitio, franja de altitud, riqueza de árboles, abundancia de árboles y el área basal de los árboles. A partir de los modelos estimados, usando la distribución de Poisson, se seleccionaron las variables que más contribuyeron a la explicación del modelo de acuerdo con $p$ en un modelo saturado, descartamos las variables que menos contribuyeron a la variable respuesta. Para esto, se emplearon modelos considerando los interceptos y los diferentes modelos a partir de la combinación de las covariables, para lo cual se tomó el valor del criterio de Akaike AICc, la diferencia entre los modelos Delta AICc, el peso de cada modelo AICc WT y su acumulado Cum. WT; lo anterior mediante el lenguaje de programación R (RStudio Team, 2020).

\section{Resultados}

\subsection{Comunidades de plantas}

En los 72 transectos de vegetación se encontraron 1147 árboles, 90 especies pertenecientes a 36 familias. La representatividad promedio de árboles en todos los sitios fue del $83.53 \%$ (Apéndice 1). La mayor abundancia de árboles por transecto se encontró en el bosque ripario bajo (21 individuos), mientras que el menor estuvo en el matorral de la franja media (9 individuos). En cuanto a la riqueza el mayor valor estuvo en el bosque ripario de la franja media (16 especies) y el menor en los matorrales de la franja baja (7 especies), encontrando valores intermedios entre los diferentes tipos de vegetación.

En todos los sitios muestreados, las familias con mayor riqueza fueron: Fabaceae (20 especies), Myrtaceae (7 especies), Malvaceae, Moraceae, Melastomataceae (5 especies), Euphorbiaceae y Apocynaceae (4 especies). Las familias con mayor número de individuos fueron Fabaceae (375 individuos), Anacardiaceae (125 individuos), Myrtaceae (76 individuos), Moraceae (72 individuos) y Myrsinaceae (54 individuos) (Apéndice 2). La mayor riqueza de plantas se obtuvo en el bosque ripario y el matorral de la franja media. Mientras que la menor se encontró en los matorrales de la franja alta y baja (Figura 3).

\begin{tabular}{|c|c|c|c|c|c|}
\hline 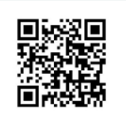 & (c) (i) () () & $\underset{\text { AMBIINTALS }}{\infty}$ & 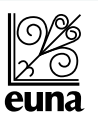 & 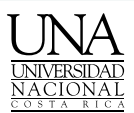 & 205 \\
\hline
\end{tabular}




\section{Revista de CIENCIAS AMBIENTALES Tropical Journal of Environmental Sciences}

Revista de Ciencias Ambientales (Trop J Environ Sci) e-ISSN: 2215-3896

(Julio-Diciembre, 2021) . Vol 55(2): 197-228 DOI: https://doi.org/10.15359/rca.55-2.10 Open Access: www.revistas.una.ac.cr/ambientales e-mail: revista.ambientales@una.ac.cr Zuluaga-Carrero J. y Renjifo L.
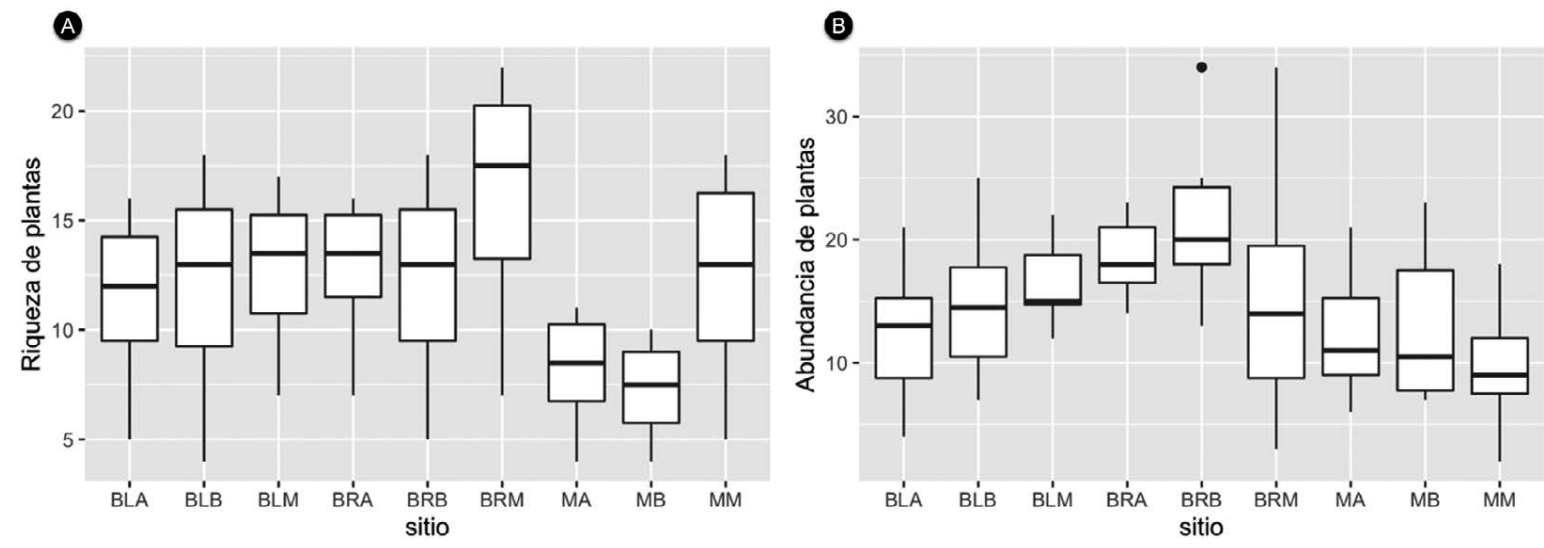

Figura 3. A. Riqueza y B. abundancia de árboles en el enclave seco del cañón río del Chicamocha, Colombia. La riqueza se obtuvo a partir de la rarefacción y la abundancia tomando el número de árboles, ambos por transecto. Figure 3. A. Trees richness and B. abundance in the dry enclave of Chicamocha river canyon, Colombia. Richness was obtained from rarefaction and abundance taking the number of trees per transect.

El índice de similaridad de Bray-Curtis construyó dos grupos para los sitios, de acuerdo con su fisionomía, los matorrales con un $50 \%$ y los bosques con un $51 \%$, así como dos grupos más para las franjas de altitud, uno conformado por la franja media y baja con un $72 \%$, y otro para la franja alta con $94 \%$ de similitud en la composición de especies de plantas. Los valores del área basal de cada tipo de vegetación mostraron diferencias significativas $\mathrm{Chi}^{2} \mathrm{~F}=7,645 P=0,044$ $\mathrm{gl}=8$. La mayor área basal promedio la tiene el bosque ripario de la franja altitudinal media $\left(10.7 \pm 6,0 \mathrm{~m}^{2} / \mathrm{ha}\right)$, un valor intermedio en el bosque de ladera de la franja baja $\left(4.7 \pm 3.3 \mathrm{~m}^{2} / \mathrm{ha}\right)$ y el más bajo en los matorrales de la franja alta $\left(0.4 \pm 0.2 \mathrm{~m}^{2} / \mathrm{ha}\right)$. Por tanto, hay diferencias en la composición de la vegetación de acuerdo con la franja altitudinal, mientras que las diferencias estructurales se observan de acuerdo a las fisionomías de vegetación.

\subsection{Comunidades de aves}

Obtuvimos 2702 registros en total, durante los conteos correspondientes, de 103 especies distribuidas en 27 familias. Las familias con el mayor número de especies en todos los sitios fueron Tyrannidae (19 especies), Thraupidae (18 especies), Emberizidae (8 especies), Cuculidae, Icteridae y Parulidae (6 especies) (Apéndice 4). Entre estas familias de aves se incluyen especies que se encuentran en alguna categoría de amenaza, como por ejemplo: Saucerottia castaneiventris (EN-VU), Thryophilus nicefori (CR-EN-VU), Arremon schlegeli (VU) e Icterus icterus (VU) (Renjifo et al., 2017). Se observaron, además, de las dos especies anteriormente mencionadas, otras especies endémicas para el país según Chaparro-Herrera et al. (2013), entre ellas Ortalis columbiana, Saucerottia cyanifrons y Myiarchus apicalis. Así como también especies casi endémicas como Forpus conspicillatus, Chlorostilbon gibsoni, Chlorostilbon poortmani, Thamnophilus multistriatus, Ramphocelus dimidiatus, Stilpnia vitriolina y Arremon atricapillus.

\begin{tabular}{|c|c|c|}
\hline 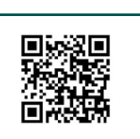 & (c) (i) (5)(2) & 206 \\
\hline
\end{tabular}




\section{Revista de CIENCIAS AMBIENTALES Tropical Journal of Environmental Sciences}

Revista de Ciencias Ambientales (Trop J Environ Sci) e-ISSN: 2215-3896

(Julio-Diciembre, 2021). Vol 55(2): 197-228 DOI: https://doi.org/10.15359/rca.55-2.10 Open Access: www.revistas.una.ac.cr/ambientales e-mail: revista.ambientales@una.ac.cr Zuluaga-Carrero J. y Renjifo L.

Los estimadores no paramétricos mostraron valores cercanos al número de especies observado, con una representatividad del $94 \%$ en promedio para todos los tipos de vegetación muestreados (Apéndice 3). Hay diferencias en la riqueza y abundancia entre sitios; la fisionomía que mostró mayor riqueza de especies fue los matorrales, los bosques de ladera y el bosque ripario de la franja baja (Figura 4). El mayor número de especies se observó en los matorrales (30 especies), mientras que el bosque ripario (23 especies) y los bosques de ladera (22 especies). Por otra parte, la abundancia media observada mostró variaciones entre los sitios; el mayor fue el matorral de la franja media (11 individuos) y el menor el bosque ripario de la franja baja (5 individuos).
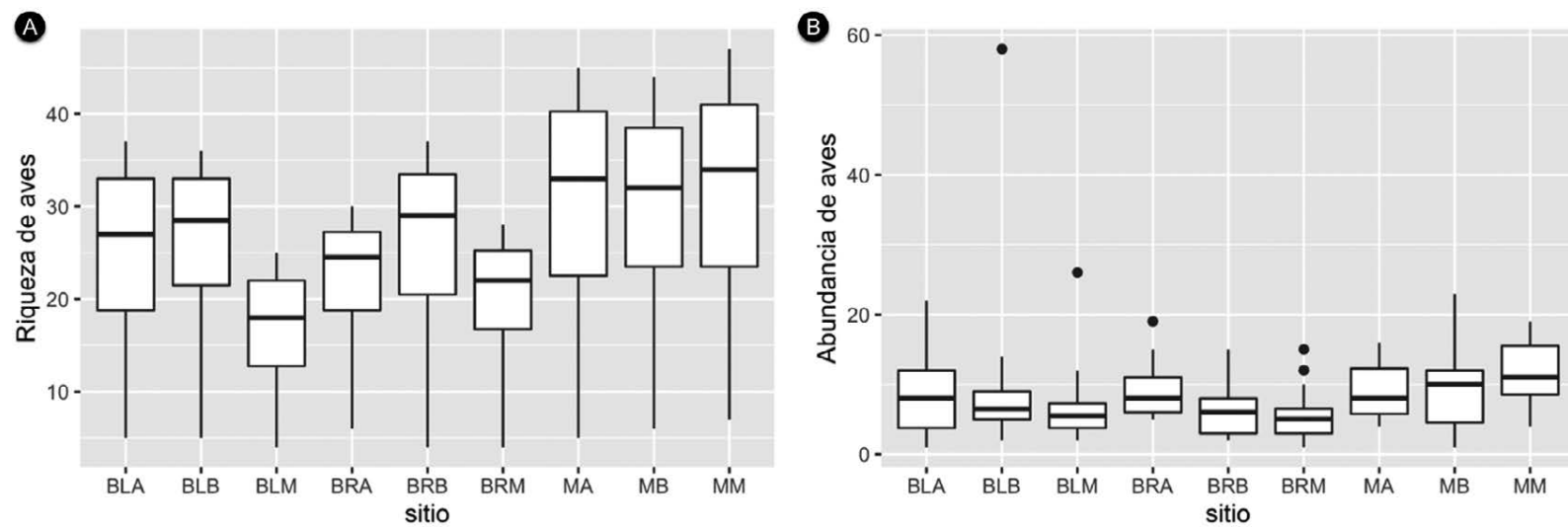

Figura 4. A. Riqueza y B. abundancia de aves en el enclave seco del cañón río del Chicamocha, Colombia. La riqueza se obtuvo a partir de la rarefacción y la abundancia tomando la media de individuos por transecto.

Figure 4. A. Bird richness and B. abundance in the dry enclave of Chicamocha river canyon, Colombia. Richness was obtained from rarefaction and abundance using mean per transect.

Tanto la riqueza como la abundancia de aves varía entre sitios, cambios que además se relacionan con la franja de altitud. La riqueza estimada fue similar en los bosques, aunque los mayores valores se observaron en los matorrales, el bosque ripario de la franja baja y el bosque de ladera de la franja baja. Se encontraron diferencias en la abundancia por especie de aves en cada sitio. Tan solo 66 de las 106 especies de aves observadas contaron con registros suficientes para el análisis con 10 registros por tipo de vegetación (Cuadro 1). A partir de estos análisis se encontró que un $15 \%$ de las 60 especies de aves posee diferencias significativas en el número de individuos entre los tipos de vegetación muestreados. Entre estas especies se deben resaltar aquellas que se observaron solo en bosque (ripario y ladera), tal como Amazona ochrocephala, Dendroplex picus y Megarynchus pitangua, así como también aquellas especies que se observaron solo en los matorrales como Colinus cristatus, Columbina talpacoti, Pyrocephalus rubinus, Todirostrum cinereum, Thraupis episcopus y Volatinia jacarina.

\begin{tabular}{|c|c|c|}
\hline 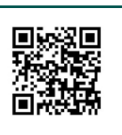 & (c) (i) (2)(2) & 207 \\
\hline
\end{tabular}




\section{Revista de CIENCIAS AMBIENTALES Tropical Journal of Environmental Sciences}

Revista de Ciencias Ambientales (Trop J Environ Sci) e-ISSN: 2215-3896

(Julio-Diciembre, 2021) . Vol 55(2): 197-228

DOI: https://doi.org/10.15359/rca.55-2.10

Open Access: www.revistas.una.ac.cr/ambientales e-mail: revista.ambientales@una.ac.cr Zuluaga-Carrero J. y Renjifo L.

Cuadro 1. Listado de las especies que mostraron diferencias significativas en el número de individuos entre tipos de vegetación en el enclave seco del cañón río del Chicamocha, Colombia. A cada especie se le asignó además la fisionomía dominante en la que fueron observadas en este estudio. El valor de probabilidad corresponde a la prueba no paramétrica de Friedman $p>0,05$.

Table 1. List of species with significant differences in the number of individuals between types of vegetation in the dry enclave of the Chicamocha river canyon, Colombia. Each species was also assigned the dominant physiognomy in which they were observed in this study. The probability value corresponds to the non-parametric Friedman test $\mathrm{p}>0.05$.

\begin{tabular}{lllll}
\hline \multicolumn{1}{c}{ Orden } & \multicolumn{1}{c}{ Familia } & \multicolumn{1}{c}{ Especie } & Probabilidad & \multicolumn{1}{c}{ Fisionomía } \\
\hline GALLIFORMES & Odontophoridae & Colinus cristatus & 0.007 & Matorral \\
ACCIPITRIFORMES & Accipitridae & Rupornis magnirostris & 0.007 & Bosque y matorral \\
COLUMBIFORMES & Columbidae & Columbina talpacoti & 0.027 & Matorral \\
CUCULIFORMES & Cuculidae & Tapera naevia & 0.000 & Bosque y matorral \\
PSITTACIFORMES & Psittacidae & Amazona ochrocephala & 0.015 & Bosque \\
PASSERIFORMES & Furnariidae & Dendroplex picus & 0.006 & Bosque \\
& Tyrannidae & Pyrocephalus rubinus & 0.021 & Matorral \\
& & Todirostrum cinereum & 0.020 & Matorral \\
& & Myiozetetes cayanensis & 0.025 & Bosque y matorral \\
& & Pitangus sulphuratus & 0.055 & Bosque y matorral \\
& & Megarynchus pitangua & 0.055 & Bosque \\
& Thraupidae & Thraupis episcopus & 0.000 & Matorral \\
& & Stilpnia vitriolina & 0.000 & Bosque y matorral \\
& & Saltator striatipectus & 0.000 & Bosque y matorral \\
& & Volatinia jacarina & 0.007 & Matorral \\
& & Arremon schlegeli & 0.000 & Bosque y matorral \\
\hline
\end{tabular}

La distribución de abundancia de las comunidades se ajustó a dos modelos de distribución, el primero, vara partida, y, el segundo, a serie logarítmica (Apéndice 5 y 6). Las comunidades de la franja alta tiene mejor ajuste al modelo de vara partida. Por otra parte, las comunidades de aves de los tres tipos de vegetación de la franja baja se ajustaron al modelo de serie logarítmica. Finalmente, las comunidades de la franja media varían con serie logarítmica en el bosque de ladera y el matorral y vara partida en el bosque ripario.

Los sitios que presentaron los menores valores fueron los bosques (ripario y ladera) de la franja baja, mientras que los tipos de vegetación que presentaron la mayor diversidad de aves fueron los matorrales de la franja alta. Se resalta la baja diversidad que mostraron los bosques riparios (entre 32-42 especies). Así mismo, los bosques de ladera mostraron una diversidad intermedia entre los matorrales y los bosques riparios (entre 38-53 especies), siendo los matorrales los que cuentan con mayor diversidad de aves (entre 47-55 especies). A partir del análisis de agrupamiento de aves se obtuvieron dos grupos, el primero para las especies de bosque y el

\begin{tabular}{|c|c|c|c|c|}
\hline 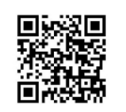 & (c) (1) & $\underset{A M B E N \text { NAILIES }}{\infty}$ & $\frac{1 \%}{\text { euna }}$ & 208 \\
\hline
\end{tabular}




\section{Revista de CIENCIAS AMBIENTALES Tropical Journal of Environmental Sciences}

Revista de Ciencias Ambientales (Trop J Environ Sci) e-ISSN: 2215-3896

(Julio-Diciembre, 2021) . Vol 55(2): 197-228

DOI: https://doi.org/10.15359/rca.55-2.10 Open Access: www.revistas.una.ac.cr/ambientales e-mail: revista.ambientales@una.ac.cr Zuluaga-Carrero J. y Renjifo L. segundo para las especies de matorrales (Figura 5A), con una similitud en la composición entre franjas altitudinales (Figura 5B).

Se obtuvieron valores para el índice de Williams entre 0.1 y 0.3 , siendo este último el valor más alto de recambio de especies (Apéndice 7). Las mayores diferencias se encuentran entre los bosques riparios de la franja alta y los matorrales de la franja baja $(\beta-3=0.356)$, mientras que hay un bajo recambio en los bosques riparios de la franja alta y la franja baja $(\beta-3=0.192)$. Igualmente, se presenta mayor variación entre bosques y matorrales, por ejemplo, encontrando cambios entre la franja alta y la baja.

\subsection{Comunidades de aves por sitios}

A partir del análisis de escalamiento multidimensional se analizaron las afinidades de las especies de aves con diferentes sitios. Las comunidades de aves se agruparon en dos bosque y matorrales (Figura 6), compartiendo especies entre franjas altitudinales.

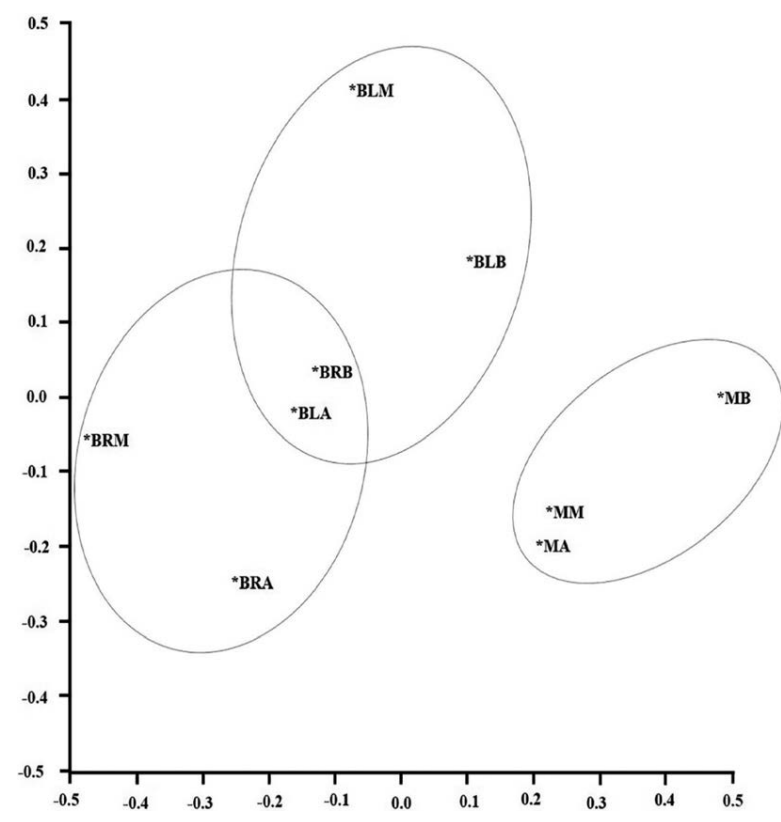

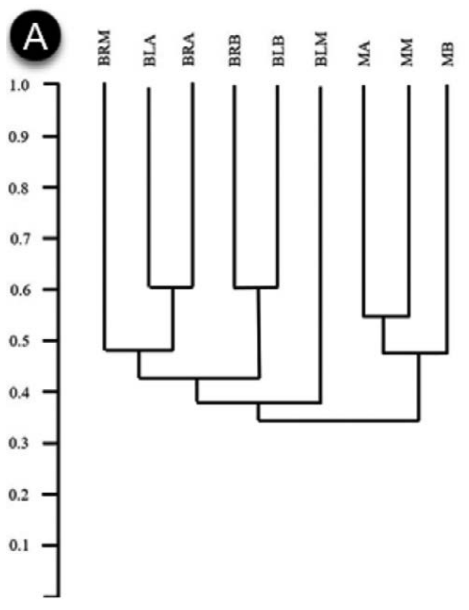

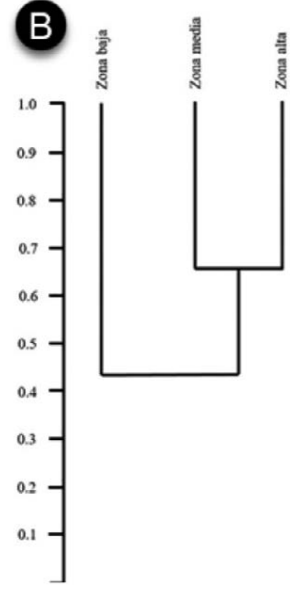

Figura 5. Dendrogramas de similitud Bray-Curtis especies de aves entre sitios de muestreo en el enclave seco del cañón río del Chicamocha, Colombia. A) Similaridad de las comunidades de aves en cada uno de los tipos de vegetación y B) Similaridad de las comunidades de aves por franja de altitud.

Figure 5. Dendrograms of similarity Bray-Curtis bird species between sampling sites in the dry enclave of the Chicamocha river canyon, Colombia. A) Similarity of bird communities in each type of vegetation and $\mathbf{B}$ ) Similarity of the bird communities by altitude stripe.

Figura 6. Análisis de escalamiento multidimensional (nMDS) de las especies de aves y sus afinidades en nueve sitios en el enclave seco del cañón del río Chicamocha, Colombia. En el análisis se muestra que los sitios tienen similaridad en la composición de sus comunidades de acuerdo con la fisionomía de vegetación, principalmente, encontrando dos grupos, las especies de bosques (riparios $\mathrm{BR}$ y laderas $\mathrm{BL}$ ) y las especies de los matorrales $\mathrm{M}$, en la franja alta A, media M y baja B; en este análisis de ordenación fueron utilizadas distancias euclidianas.

Figure 6. Multidimensional scaling analysis (nMDS) of bird species and their affinities in nine sites in the dry enclave of the Chicamocha river canyon, Colombia. The analysis shows that the sites have similarity in the composition of their communities according to the physiognomy of vegetation mainly, finding two groups, the forest species (BR riparian and $\mathrm{BL}$ slopes) and the $\mathrm{M}$ scrub species, In the high band $\mathrm{A}$, medium $\mathrm{M}$ and low $\mathrm{B}$, in this ordering analysis Euclidean distances were used.

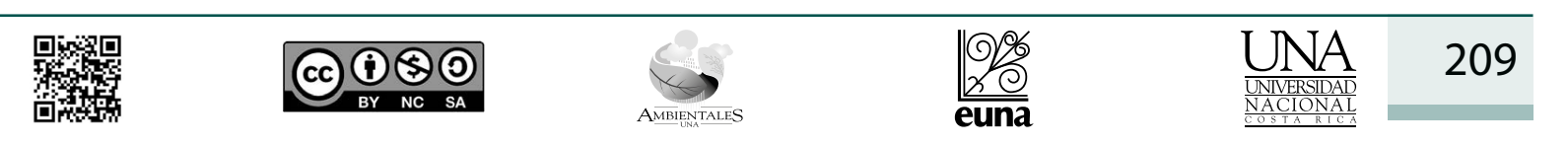




\section{Revista de CIENCIAS AMBIENTALES Tropical Journal of Environmental Sciences}

Revista de Ciencias Ambientales (Trop J Environ Sci) e-ISSN: 2215-3896

(Julio-Diciembre, 2021) . Vol 55(2): 197-228

DOI: https://doi.org/10.15359/rca.55-2.10

Open Access: www.revistas.una.ac.cr/ambientales e-mail: revista.ambientales@una.ac.cr Zuluaga-Carrero J. y Renjifo L.

De manera adicional a los análisis anteriores, se presenta un modelo con el cual se evaluaron dos variables independientes, riqueza y abundancia de aves, a partir de las variables dependientes de la vegetación establecidas. De este modo, mediante un modelo saturado, se obtuvieron las variables significativas que aportan a la explicacións (Cuadro 4).

Cuadro 4. Modelos lineares generalizados. Se presentan diferentes modelos con una combinación de variables independientes para cada variable dependiente del mejor modelo en el enclave seco del cañón del río Chicamocha, Colombia. Se seleccionaron los mejores modelos basados en el menor valor del criterio de Akaike AICc, la diferencia entre los modelos Delta AICc, el valor de explicación de cada modelo AICc WT y el acumulado de la explicación Cum. WT.

Table 4. Generalized linear models. Different models are presented with a combination of independent variables for each response variable, for the best model in the dry enclave of the Chicamocha river canyon, Colombia. Models were selected based on the lowest value of the Akaike criterion AICc, the difference between models Delta AICc, explanation value of each model AICc WT and the cumulative explanation of them Cum. WT.

\begin{tabular}{|c|c|c|c|c|}
\hline Variables dependientes & AICc & Delta AICc & AICc Wt & Cum. Wt \\
\hline \multicolumn{5}{|l|}{ Abundancia de las aves } \\
\hline Abundancia aves $\sim$ Sparboles + Sitio + SpAves & 20.79 & 0 & 0.44 & 0.44 \\
\hline Abundancia aves $\sim$ Altura + Sparboles + Sitio + AB + SpAves & 20.79 & 0 & 0.44 & 0.88 \\
\hline Abundancia aves $\sim$ Altura + Sparboles + Sitio + Abarboles + AB & 23.46 & 2.67 & 0.12 & 1 \\
\hline \multicolumn{5}{|l|}{ Riqueza de las aves } \\
\hline Riqueza aves $\sim$ Sparboles + Sitio + Abaves & 388.31 & 0 & 0.45 & 0.45 \\
\hline Riqueza aves $\sim$ Altura + Sparboles + Sitio + Abaves & 391.31 & 0 & 0.45 & 0.9 \\
\hline Riqueza aves $\sim$ Altura + Sparboles + Sitio $+\mathrm{AB}+$ Abaves & 393.29 & 2.98 & 0.1 & 1 \\
\hline
\end{tabular}

Las variables dependientes que mayor aportan a la explicación de las variables respuesta varían mostrando valores significativos en la variable dependiente (Cuadro 4). La abundancia de aves se puede explicar mejor a partir del sitio, la franja de altitud y la abundancia de árboles, mientras que la riqueza de aves puede explicarse mejor a partir del sitio y la franja de altitud. Finalmente, el área basal $\mathrm{AB}$ no brindó aportes relevantes en la explicación de los modelos.

\section{Discusión}

Se registraron 90 especies de plantas, pertenecientes a 36 familias en el área de estudio, lo cual representa un $62 \%$ de las especies arbóreas y arbustivas reportadas para el cañón del río Chicamocha (Albesiano \& Rangel-Ch, 2006). Se destacan especies de árboles por su abundancia en algunas franjas altitudinales, aunque no hay diferencias entre tipos de vegetación en el número de especies. Es probable que, al incluir otras formas de crecimiento que tomen diámetros a la altura del pecho con menores valores, el porcentaje de especies se incremente, particularmente al incluir especies herbáceas que, además, varían con la estacionalidad en el bosque seco tropical (Albesiano \& Fernández-Alonso, 2006).

\begin{tabular}{|c|c|c|c|c|}
\hline 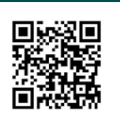 & (c) (i) (\$) & $\underset{\text { AMBIENTALIS }}{S}$ & $\frac{1 \%}{\frac{\%}{2}}$ & 210 \\
\hline
\end{tabular}




\section{Revista de CIENCIAS AMBIENTALES Tropical Journal of Environmental Sciences}

Revista de Ciencias Ambientales (Trop J Environ Sci)

e-ISSN: 2215-3896

(Julio-Diciembre, 2021) . Vol 55(2): 197-228

DOI: https://doi.org/10.15359/rca.55-2.10

Open Access: www.revistas.una.ac.cr/ambientales e-mail: revista.ambientales@una.ac.cr Zuluaga-Carrero J. y Renjifo L.

La composición de especies de aves obtenida en este estudio representa un $76 \%$ de los registros que otros estudios obtuvieron en bosques secos tropicales (Losada-Prado \& Molina-Martínez, 2011) y un 25 \% más que el registrado en bosques transicionales con bosque seco del zonobioma húmedo (Sáenz et al., 2006). De igual manera, en el presente estudio se obtiene un $17 \%$ más de las especies observadas por Collazos-González \& Echeverry-Galvis, (2017) y un 35 $\%$ de lo reportado para la región del cañón del río Chicamocha (Collazos-González et al., 2020).

Nuestros resultados indican diferencias significativas entre franjas altitudinales con un mayor número de especies de aves en la franja alta y un menor número en la franja baja. Algunos estudios en bosques húmedos señalan que la riqueza de especies disminuye con la altitud y que hay diferencias en la composición de las comunidades de aves, explicando estas variaciones por cambios en el área de bosque, disminución de invertebrados y variaciones en las condiciones ambientales (Blake \& Loiselle, 2000). Sin embargo, nuestros resultados muestran que este patrón no se presenta en el bosque seco, variaciones que pueden explicarse por una mayor disponibilidad de agua (Poulin et al., 1993), observable durante la temporada de lluvias.

También encontramos cambios diferenciales al analizar los sitios por separado en el gradiente de altitud. Los bosques de ladera son más diversos al compararse con los bosques riparios, mientras que los matorrales son los que cuentan con la mayor riqueza de especies. Algunos autores destacan el valor de los bosques riparios como corredores clave para las aves en bosque seco, al funcionar como piedras de paso entre coberturas de vegetación (Gillies et al., 2011) y que, además, su mayor complejidad estructural puede ser una característica relevante en el establecimiento de áreas protegidas (Bennett et al., 2014). De manera contraria, en este estudio encontramos que los bosques riparios tienen una menor riqueza y que los bosques de ladera, sorprendentemente, albergan la mayor parte de las especies de bosque, resultados que pueden explicarse por la mayor diversidad florística de los bosques de ladera. Así mismo, es posible que este resultado se relacione con la transformación humana de la vegetación y la escasez de agua observada en estos estos bosques (Jansen \& Robertson, 2001). Llama la atención que los bosques de ladera, por ejemplo, estén en áreas de mayor pendiente y con un acceso más limitado para actividades humanas y que tengan una mayor diversidad florística y de aves, características que en este caso son relevantes para establecer un área protegida, tomando en consideración las laderas.

Se encontraron especies que comparten fisionomías de vegetación o que están restringidas a una sola. Se identifican dos grupos principales en las aves que, en este caso, comparten especies; el primer grupo, las aves de bosque, en el que se encuentran tanto aves de los bosques riparios como las de los bosques de ladera; en un segundo grupo se incluye solo las especies de los matorrales. Del total de especies analizadas, tan solo un $11 \%$ contaron con registros únicos para una fisionomía de vegetación (bosques riparios, bosques de ladera o matorrales), por lo que la mayoría de las especies analizadas comparten fisionomías de vegetación. Un $67 \%$ de las aves de bosque comparten tanto bosques de ladera como riparios, mientras que un $33 \%$ solo se ubican en los matorrales. Entre las especies que se observaron en ambos bosques (ripario y ladera) se encuentra Dendroplex picus y Megarynchus pitangua, así como también especies que

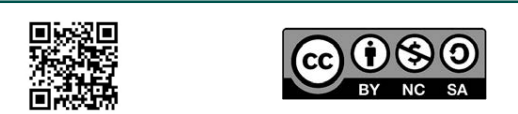

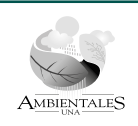




\section{Revista de CIENCIAS AMBIENTALES Tropical Journal of Environmental Sciences}

Revista de Ciencias Ambientales (Trop J Environ Sci)

e-ISSN: 2215-3896

(Julio-Diciembre, 2021) . Vol 55(2): 197-228

DOI: https://doi.org/10.15359/rca.55-2.10

Open Access: www.revistas.una.ac.cr/ambientales e-mail: revista.ambientales@una.ac.cr Zuluaga-Carrero J. y Renjifo L.

se observaron solo en los matorrales como Colinus cristatus, Columbina talpacoti, Pyrocephalus rubinus, Todirostrum cinereum, Thraupis episcopus y Volatinia jacarina. Distribución que coincide con lo señalado por Khan (2017), quien menciona que en áreas abiertas como matorrales hay un mayor número de individuos de especies generalistas, principalmente, mientras que, en áreas boscosas, hay mayor densidad de especialistas y una alta diversidad de árboles, registros que concuerdan con lo observado por otros autores en bosque seco (Losada-Prado \& Molina-Martínez, 2011; Santamaría-Rivero et al., 2016).

La distribución de la abundancia de las especies de aves en la franja alta sugiere una mayor disponibilidad de recursos, mientras que, en la franja baja, la comunidad tiene una mayor proporción de especies raras y una menor cantidad de especies abundantes, sugiriendo menor cantidad de recursos. Al comparar los registros de especies más abundantes, se observaron especies similares a las registradas en bosque seco (Collazos-González et al., 2020; Collazos-González \& Echeverry-Galvis, 2017; Losada-Prado \& Molina-Martínez, 2011), entre las que se incluyen Saltator striatipectus, Pitangus sulphuratus, Sporophila minuta, así como también especies con distribuciones más restringidas que pueden observarse en la región del cañón del río Chicamocha, como Saucerottia castaneiventris, Myiarchus apicalis y Arremon schlegeli. Es posible que las condiciones ambientales más secas y extremas de la franja baja sean las que están estructurando las comunidades de aves. Collazos-González \& Echeverry-Galvis (2017) observaron un patrón de distribución logarítmico para la zona de estudio y resaltan que, en los bosques secos tropicales particularmente, los cambios climáticos llevan a cambios fenológicos, limitando así la disponibilidad de recursos como la vegetación e invertebrados, por ejemplo, en el que las aves raras estarían susceptibles a desaparecer ya que sus requerimientos estarían pobremente satisfechos. Resulta indispensable, por lo tanto, entender las variaciones en la comunidad en el largo plazo con el propósito de comprender cómo influye la estacionalidad en las aves de las zonas más bajas.

En este estudio se observamos la forma en la que se comparten especies entre fisionomías de vegetación en todo el gradiente altitudinal, en el que además hay heterogeneidad en la composición florística. En gradientes altitudinales, la diversidad del hábitat puede explicar los cambios en la riqueza de las especies, encontrando que algunas especies tienen distribuciones restringidas a un tipo de vegetación, mientras otras cuentan con distribuciones más amplias, lo que está relacionado sus requerimientos de hábitat, especies con distribuciones restringidas a un tipo de vegetación particular, pueden tener requerimientos de hábitat más estrechos, por ejemplo las aves especialistas (Kim et al., 2019). Así, la franja alta cuenta con especies de árboles que corresponden con vegetación de bosque seco correspondiente al orobioma azonal del zonobioma húmedo tropical, mientras que la franja baja cuenta con especies de árboles del zonobioma alternohígrico tropical, sin embargo, en el bosque de ladera se observaron especies de aves a través de toda la franja de altitud, compartiendo especies a través de toda la franja de altitud del enclave seco.

\begin{tabular}{|c|c|c|c|c|c|}
\hline 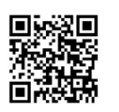 & (c) (i) $\$\left(\begin{array}{l}\mathrm{BY} \\
\mathrm{NC}\end{array}\right.$ & 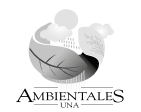 & $\frac{O \%}{2 \%}$ & $\frac{\text { UNA }}{\frac{\text { UNNVERSIDAD }}{\frac{N A C I O N A L}{\text { NASTARACA }}}}$ & 212 \\
\hline
\end{tabular}




\section{Revista de CIENCIAS AMBIENTALES Tropical Journal of Environmental Sciences}

Revista de Ciencias Ambientales (Trop J Environ Sci)

e-ISSN: 2215-3896

(Julio-Diciembre, 2021) . Vol 55(2): 197-228

DOI: https://doi.org/10.15359/rca.55-2.10

Open Access: www.revistas.una.ac.cr/ambientales e-mail: revista.ambientales@una.ac.cr Zuluaga-Carrero J. y Renjifo L.

La heterogeneidad florística puede ser un factor importante en la distribución amplia de ciertas especies de aves a través de este gradiente. Aunque en este estudio no se observaron aves restringidas a una única franja de altitud, la familia Icteridae mostró mayor diversidad en la franja baja en comparación a la franja alta. Otros estudios han demostrado que ciertas especies de flora pueden favorecer la presencia de algunas especies de aves (Almazán-Núñez et al., 2016). En este estudio la continuidad de la fisionomía de la vegetación podría estar brindando áreas continuas de bosque, un requerimiento clave para esta familia. Una alta diversidad florística favorece la abundancia de aves, aportando recursos para sostener comunidades más diversas, como se ha observado en otros bosques secos que ocurren en paisajes agroforestales (Sáenz et al., 2006). Por lo cual, sugerimos que en futuros estudios se identifique cuáles especies de plantas son claves para la avifauna y su aporte a la interacción entre fisionomías de vegetación, poniendo especial atención para aquellas especies que se encuentren amenazadas o que son endémicas de los bosques secos tropicales.

No se observó una relación clara entre las variables de estructura de la vegetación seleccionadas y la respuesta de las aves al gradiente altitudinal. La complejidad estructural es una de las variables que tiene un aporte importante para las aves (Sitters et al., 2016), atribuyendo a estas variables una mayor cantidad de especies de aves favorecidas por la diversidad de recursos como alimento, percha y nidificación, entre otros (Corcuera \& Zavala-Hurtado, 2014). No obstante, al relacionar el área basal con el número de especies y el número de individuos de aves entre sitios, no hubo un aporte significativo a la hipótesis planteada y el efecto que puede tener la estructura de la vegetación en la diversidad de avifauna. Por lo tanto, se sugiere evaluar otras variables de estructura de la vegetación, como estratos de vegetación que no fueron incluidos en este estudio y podrían brindar la respuesta de la presencia de ciertas aves en el gradiente altitudinal (Müller et al., 2010). De igual manera, se sugiere incluir otras formas de vida de vegetación, como herbáceas, arbustos y epífitas, pues estas plantas podrían tener un aporte de vital importancia en la dinámica estacional de los bosques secos tropicales, en los cuales hay especies que cambian notablemente su cobertura y oferta de recursos entre la estación seca y la estación lluviosa (Pizano \& García, 2014).

Los bosques secos tropicales cuentan con una amplia variación de vegetación que les brindan a las aves una alta diversidad de recursos, los que, además, pueden variar con la estacionalidad en las lluvias. En este estudio observamos cambios en la riqueza y abundancia de las aves en distintas fisionomías en un gradiente altitudinal. Aunque los bosques riparios sean los que merezcan una mayor atención, por su posible transformación antrópica, y que en este estudio se asocia algunas especies a una fisionomía de vegetación particular. En el presente trabajo resulta notable el aporte de los bosques de ladera por su diversidad florística a través del gradiente altitudinal, una fisionomía que podría ser priorizada en el establecimiento de áreas protegidas, a partir de las cuales sea posible brindar continuidad y diversidad de recursos para la conservación de las especies de aves de bosque seco tropical.

\begin{tabular}{|c|c|c|c|c|c|}
\hline 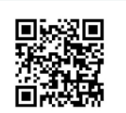 & (c) (1) $\$$ & $\underbrace{}_{\text {AMBIFNAIIES }}$ & $\begin{array}{l}\frac{9 \%}{2} \\
\frac{2}{2} \\
\text { euna }\end{array}$ & 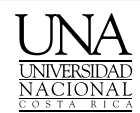 & 213 \\
\hline
\end{tabular}




\section{Revista de CIENCIAS AMBIENTALES Tropical Journal of Environmental Sciences}

Revista de Ciencias Ambientales (Trop J Environ Sci)

e-ISSN: 2215-3896

(Julio-Diciembre, 2021). Vol 55(2): 197-228

DOI: https://doi.org/10.15359/rca.55-2.10

Open Access: www.revistas.una.ac.cr/ambientales e-mail: revista.ambientales@una.ac.cr Zuluaga-Carrero J. y Renjifo L.

\section{5. Ética y conflicto de intereses}

Las personas autoras declaran que han cumplido totalmente con todos los requisitos éticos y legales pertinentes, tanto durante el estudio como en la producción del manuscrito; que no hay conflictos de intereses de ningún tipo; que todas las fuentes financieras se mencionan completa y claramente en la sección de agradecimientos; y que están totalmente de acuerdo con la versión final editada del artículo.

\section{Agradecimientos}

Esta investigación fue financiada por la beca otorgada por la Fundación Conserva, que hizo parte del convenio de colaboración N. 5212094 entre ECOPETROL, Fundación Estación Biológica Guayacanal, Fundación Chimbilako y Fundación Conserva. Este estudio se realizó con el permiso de colecta No. 0284 otorgado por la Autoridad Nacional de Licencias Ambientales. Agradecemos a todos aquellos que participaron como asistentes de campo a: Javier Ayala, Cecilia García y a Holman Durán, y al apoyo de Alexandra Delgadillo y a Germán Camargo por el apoyo durante la fase de campo. Y finalmente, a los evaluadores del manuscrito, por sus valiosas contribuciones.

\section{Referencias}

Albesiano, S., Fernández-Alonso, J. L. (2006). Catálogo comentado de la flora vascular de la franja tropical (500-1200m) del cañón del río Chicamocha (Boyacá-Santander, Colombia). Primera parte. Caldasia, 28(1), 23-44.

Albesiano, S., Rangel-Ch, J. O. (2006). Estructura de la vegetación del Cañón del río Chicamocha, 500-1200m; Santander-Colombia: Una herramienta para la conservación. Caldasia, 28(2), 307-325.

Almazán-Núñez, R. C., Eguiarte, L. E., Arizmendi, M. del C., Corcuera, P. (2016). Myiarchus flycatchers are the primary seed dispersers of Bursera longipes in a Mexican dry forest. PeerJ, 4, e2126. https://doi.org/10.7717/peerj.2126

Anderson, M. J., Crist, T. O., Chase, J. M., Vellend, M., Inouye, B. D., Freestone, A. L., Sanders, N. J., Cornell, H. V., Comita, L. S., Davies, K. F., Harrison, S. P., Kraft, N. J. B., Stegen, J. C., Swenson, N. G. (2011). Navigating the multiple meanings of $\beta$ diversity: A roadmap for the practicing ecologist: Roadmap for beta diversity. Ecology Letters, 14(1), 19-28. https://doi. org/10.1111/j.1461-0248.2010.01552.x

Avalos, G., \& Mulkey, S. S. (1999). Seasonal Changes in Liana Cover in the Upper Canopy of a Neotropical Dry Forest1. Biotropica, 31(1), 186-192. https://doi.org/10.1111/j.1744-7429.1999.tb00130.x

Banda-R, K., Delgado-Salinas, A., Dexter, K. G., Linares-Palomino, R., Oliverira-Filho, A., Prado, D., Pullan, M., Quintana, C., Riina, R., Rodríguez, G. M., Weintritt, J. (2016). Plant

(c)
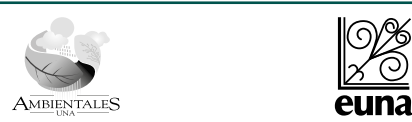


\section{Revista de CIENCIAS AMBIENTALES Tropical Journal of Environmental Sciences}

Revista de Ciencias Ambientales (Trop J Environ Sci) e-ISSN: 2215-3896

(Julio-Diciembre, 2021) . Vol 55(2): 197-228 DOI: https://doi.org/10.15359/rca.55-2.10 Open Access: www.revistas.una.ac.cr/ambientales e-mail: revista.ambientales@una.ac.cr Zuluaga-Carrero J. y Renjifo L.

diversity patterns in neotropical dry forests and their conservation implications. Science, 353(6306), 1383-1387. https://doi.org/10.1126/science.aaf5080

Bennett, A. F., Nimmo, D. G., Radford, J. Q. (2014). Riparian vegetation has disproportionate benefits for landscape-scale conservation of woodland birds in highly modified environments. Journal of Applied Ecology, 51(2), 514-523. https://doi.org/10.1111/1365-2664.12200

Blake, J. G., Loiselle, B. A. (2000). Diversity of Birds Along an Elevational Gradient in The Cordillera Central, Costa Rica. The Auk, 117(3), 663-686. https://doi.org/10.1093/auk/117.3.663

Bregman, T. P., Sekercioglu, C. H., Tobias, J. A. (2014). Global patterns and predictors of bird species responses to forest fragmentation: Implications for ecosystem function and conservation. Biological Conservation, 169, 372-383. https://doi.org/10.1016/j.biocon.2013.11.024

Brown, D. R., Sherry, T. W. (2005). Behavioral Response of Resident Jamaican Birds to Dry Season Food Supplementation1. Biotropica, 38(1), 991-999. https://doi. org/10.1111/j.1744-7429.2006.00108.x

Chaparro-Herrera, S., Echeverry-Galvis, M. Á., Córdoba-Córdoba, S., Sua-Becerra, A. (2013). Listado actualizado de las aves endémicas y casi-endémicas de Colombia. Biota Colombiana, 14(2), Article 2. http://revistas.humboldt.org.co/index.php/biota/article/view/289

Chazdon, R., L., Colwell, R., K., Denslow, J., S., Guariguata, M., R. (1998). Statistical methods for estimating species richness of woody regeneration in primary and secundary rain forest of notheastern Costa Rica. En Forest Biodiversity Research, Monitoring and Modeling. Conceptual Background and Old World Case Studies (Vol. 20). UNESCO Paris and The Parthenon Publishing Group.

Collazos-González, S. A., Echeverry-Galvis, M. Á. (2017). Comunidad de aves del bosque seco tropical en la Mesa de Xñeridas, Santander, Colombia. Ornitología Neotropical, 28, 223-235.

Collazos-González, S. A., Zuluaga-Carrero, J., Cortés-Herrera, J. O. (2020). Aves del Cañón del Chicamocha, Colombia: Un llamado para su conservación. Biota Colombiana, 21(1). https:// doi.org/10.21068/c2020.v21n01a05

Corcuera, P., Zavala-Hurtado, J. A. (2014). The influence of vegetation on bird distribution in dry forests and oak woodlands of western Mexico. Revista de Biología Tropical, 54(2), 657. https://doi.org/10.15517/rbt.v54i2.13969

Gillies, C. S., St. Clair, C. C. (2008). Riparian corridors enhance movement of a forest specialist bird in fragmented tropical forest. Proceedings of the National Academy of Sciences, 105(50), 19774-19779. https://doi.org/10.1073/pnas.0803530105

\begin{tabular}{|c|c|c|c|c|}
\hline 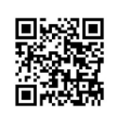 & (c) () () & $\overbrace{\text { AMBIENTIES }}$ & $\frac{1 \%}{2 \%}$ & 215 \\
\hline
\end{tabular}




\section{Revista de CIENCIAS AMBIENTALES Tropical Journal of Environmental Sciences}

Revista de Ciencias Ambientales (Trop J Environ Sci)
e-ISSN: 2215-3896
(Julio-Diciembre, 2021). Vol 55(2): 197-228
DOI: https://doi.org/10.15359/rca.55-2.10
Open Access: www.revistas.una.ac.cr/ambientales
e-mail: revista.ambientales@una.ac.cr
Zuluaga-Carrero J. y Renjifo L.

Gillies, Cameron S., Beyer, H. L., Clair, C. C. St. (2011). Fine-scale movement decisions of tropical forest birds in a fragmented landscape. Ecological Applications, 21(3), 944-954. https:// doi.org/10.1890/09-2090.1

Gotelli, N., J., Graves, G., R. (1996). Null models in Ecology. Smithsonian Institution Press.

Izsák, J., Pavoine, S. (2012). Links between the species abundance distribution and the shape of the corresponding rank abundance curve. Ecological Indicators, 14(1), 1-6. https://doi. org/10.1016/j.ecolind.2011.06.030

Jansen, A., Robertson, A. (2001). Riparian bird communities in relation to land management practices in floodplain woodlands of south-eastern Australia. Biological Conservation, 100, 173-185. https://doi.org/10.1016/S0006-3207(00)00235-4

Khan, M. S. (2017). Effect of forest composition on bird species abundance in tropical dry deciduous forest: A case of Bhimbandh Wildlife Sanctuary, India. Biodiversitas, Journal of Biological Diversity, 18(1), 78-85. https://doi.org/10.13057/biodiv/d180112

Kim, J.-Y., Seo, C., Hong, S., Lee, S., Eo, S. H. (2019). Altitudinal range-size distribution of breeding birds and environmental factors for the determination of species richness: An empirical test of altitudinal Rapoport's rule and non-directional rescue effect on a local scale. PLOS ONE, 14(1), e0203511. https://doi.org/10.1371/journal.pone.0203511

Koleff, P., Gaston, K. J., Lennon, J. J. (2003). Measuring beta diversity for presence-absence data. Journal of Animal Ecology, 72(3), 367-382. https://doi.org/10.1046/j.1365-2656.2003.00710.x

Losada-Prado, S., Molina-Martínez, Y. (2011). Avifauna del Bosque Seco Tropical en el departamento del Tolima (Colombia): Análisis de la comunidad. Caldasia, 33(1), Article 1. https:// revistas.unal.edu.co/index.php/cal/article/view/36390

McKinnon, E. A., Rotenberg, J. A., Stutchbury, B. J. M. (2015). Seasonal change in tropical habitat quality and body condition for a declining migratory songbird. Oecologia, 179(2), 363375. https://doi.org/10.1007/s00442-015-3343-1

Melo, M. A., Da Silva, M. A. G., Piratelli, A. J. (2020). Improvement of vegetation structure enhances bird functional traits and habitat resilience in an area of ongoing restoration in the Atlantic Forest. Annals of the Brazilian Academy of Sciences, 92(Supple.2), e20191241. https:// doi.org/10.1590/0001-3765202020191241

Müller, J., Stadler, J., Brandl, R. (2010). Composition versus physiognomy of vegetation as predictors of bird assemblages: The role of lidar. Remote Sensing of Environment, 114(3), 490495. https://doi.org/10.1016/j.rse.2009.10.006

Murphy, P. G., Lugo, A. E. (1987). Ecology of Tropical Dry Forest. Annual Review of Ecology and Systematics, 17, 67-88.

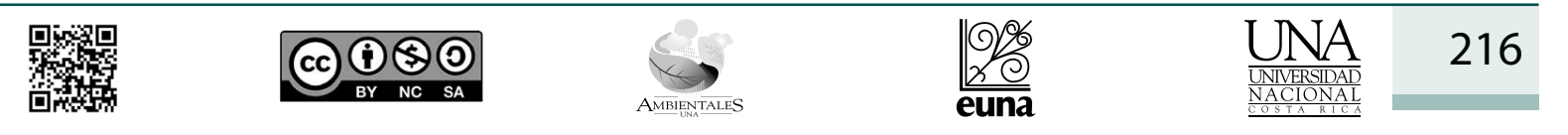




\section{Revista de CIENCIAS AMBIENTALES Tropical Journal of Environmental Sciences}

Revista de Ciencias Ambientales (Trop J Environ Sci) e-ISSN: 2215-3896

(Julio-Diciembre, 2021) . Vol 55(2): 197-228 DOI: https://doi.org/10.15359/rca.55-2.10 Open Access: www.revistas.una.ac.cr/ambientales e-mail: revista.ambientales@una.ac.cr Zuluaga-Carrero J. y Renjifo L.

Pizano, C., García, H. (Eds.). (2014). El bosque seco tropical en Colombia. Instituto de Investigación de Recursos Biológicos Alexander von Humboldt (IAvH).

Poulin, B., Lefebvre, G., McNeil, R. (1993). Variations in bird abundance in tropical arid and semi-arid habitats. Ibis, 135, 432-441. https://doi.org/10.1111/j.1474-919X.1993.tb02116.x

Ralph, C. J., Geupel, G. R., Pyle, P., Martin, T. E., DeSante, D. F., Milá, B. (1996). Manual de m\&eacute; todos de campo para el monitoreo de aves terrestres (PSW-GTR-159; p. PSW-GTR-159). U.S. Department of Agriculture, Forest Service, Pacific Southwest Research Station. https:// doi.org/10.2737/PSW-GTR-159

Renjifo, L. M., Amaya-Villarreal, Á. M., Burbano-Girón, J., Velásquez-Tibatá, J. (2017). Libro Rojo de Aves de Colombia.Volumen II. Pontificia Universidad Javeriana. http://repository. humboldt.org.co/handle/20.500.11761/34285

Rodríguez, N., Armentares, D., Morales, M., Romero, M. (2006). Ecosistemas de los andes colombianos (2. ${ }^{\mathrm{da}}$ ed.). Instituto de Investigación Alexander von Humboldt. https://www.researchgate.net/profile/Dolors-Armenteras/publication/236173774_Ecosistemas_de_los_andes_Colombianos/links/547b439c0cf205d16881c3f0/Ecosistemas-de-los-andes-Colombianos.pdf

RStudio Team. (2020). RStudio: Integrated Development for R. RStudio, PBC. http://www.rstudio.com/

Sáenz, J. C., Villatoro, F., Ibrahim, M., Fajardo, D., Pérez, M. (2006). Relación entre las comunidades de aves y la vegetación en agropaisajes dominados por la ganadería en Costa Rica, Nicaragua y Colombia. Agroforestería en las Américas, 45, 37-48.

Santamaría-Rivero, W., Leyequién, E., Hernández-Stefanoni, J. L., Wood, P. (2016). Influence of landscape structure and forest age on the richness and abundance of different bird feeding guilds and forest-dependent birds in a seasonal dry tropical forest of Yucatan, Mexico. Tropical Ecology, 57, 313-332.

Sitters, H., York, A., Swan, M., Christie, F., Di Stefano, J. (2016). Opposing Responses of Bird Functional Diversity to Vegetation Structural Diversity in Wet and Dry Forest. PLOS ONE, 11(10), e0164917. https://doi.org/10.1371/journal.pone.0164917

Vázquez, L., Moya, H., Arizmendi, M. del C. (2009). Avifauna de la selva baja caducifolia en la cañada del río Sabino, Oaxaca, México. Revista Mexicana de Biodiversidad, 80, 535-549. https://doi.org/10.22201/ib.20078706e.2009.002.626

Vázquez, L., Renton, K. (2015). High Density of Tree-Cavities and Snags in Tropical Dry Forest of Western Mexico Raises Questions for a Latitudinal Gradient. PLoS ONE, 10(1), e0116745. https://doi.org/10.1371/journal.pone.0116745

Wang, Y., Xu, J., Chen, J., Wu, B., Lu, Q. (2014). Influence of the habitat change for birds on community structure. Acta Ecologica Sinica, 34(1), 1-6. https://doi.org/10.1016/j.chnaes.2013.09.003

\begin{tabular}{|c|c|c|c|c|c|}
\hline 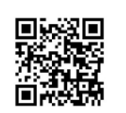 & (c) (i) (5) & $\overbrace{\text { AMBIENTIES }}$ & $\frac{10 \%}{\text { euna }}$ & $\frac{\text { UNA }}{\frac{\text { UNIVERIDAD }}{\text { NACEIONAL }}}$ & 217 \\
\hline
\end{tabular}




\section{Revista de CIENCIAS AMBIENTALES Tropical Journal of Environmental Sciences}

Revista de Ciencias Ambientales (Trop J Environ Sci) e-ISSN: $2215-3896$

(Julio-Diciembre, 2021) . Vol 55(2): 197-228

DOI: https://doi.org/10.15359/rca.55-2.10

Open Access: www.revistas.una.ac.cr/ambientales e-mail: revista.ambientales@una.ac.cr Zuluaga-Carrero J. y Renjifo L.

\section{Apéndices}

Apéndice 1. Estimadores no paramétricos de completitud de especies de árboles en cada tipo de vegetación enclave seco del Cañón del río Chicamocha, Colombia. S(obs) hace referencia a la riqueza de especies observada durante el estudio en cada transecto de vegetación; los estimadores ACE y CHAO 1 representan la riqueza estimada; el porcentaje de representatividad se calculó con el promedio entre estos dos estimadores para los ocho transectos de cada tipo de vegetación.

Appendix 1. Non-parametric estimators of completeness of tree species in each type of vegetation dry enclave of Chicamocha River Canyon, Colombia. S (obs) refers to the species richness observed during the study in each vegetation transect, the ACE and CHAO 1 estimators represent the estimated richness, the percentage of representativeness was calculated with the average between these two estimators for the eight transects of each type of vegetation.

\begin{tabular}{|c|c|c|c|c|c|c|c|c|c|c|}
\hline \multirow{10}{*}{ 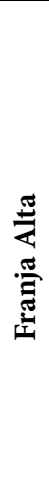 } & \multicolumn{4}{|c|}{ Bosque Ripario } & \multicolumn{3}{|c|}{ Bosque de Ladera } & \multicolumn{3}{|c|}{ Matorral } \\
\hline & Punto & S(obs) & ACE & Chao 1 & S(obs) & ACE & Chao 1 & S(obs) & ACE & Chao 1 \\
\hline & 1 & 7.5 & 14.72 & 10.5 & 5.13 & 7.12 & 6.43 & 4.38 & 7.05 & 5.13 \\
\hline & 2 & 11.96 & 18.23 & 16.68 & 8.46 & 11.82 & 11 & 6.93 & 9.17 & 7.96 \\
\hline & 3 & 14.8 & 19.53 & 17.41 & 10.91 & 13.83 & 13.17 & 8.75 & 11.56 & 10.77 \\
\hline & 4 & 16.77 & 20.42 & 18.98 & 12.9 & 15.61 & 15.24 & 10.29 & 13.22 & 12.34 \\
\hline & 5 & 18.23 & 21.63 & 20.29 & 14.63 & 16.8 & 16.47 & 11.66 & 14.94 & 13.36 \\
\hline & 6 & 19.36 & 21.88 & 20.56 & 16.18 & 17.93 & 17.68 & 12.89 & 17.01 & 14.81 \\
\hline & 7 & 20.25 & 22.33 & 21.14 & 17.63 & 18.69 & 18.34 & 14 & 18.25 & 15.96 \\
\hline & 8 & 21 & 22.41 & 21.6 & 19 & 20.1 & 19.74 & 15 & 19.2 & 16.98 \\
\hline \multirow{9}{*}{ 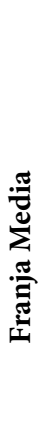 } & \multicolumn{3}{|c|}{ Representatividad } & \multicolumn{2}{|l|}{$95.46 \%$} & \multicolumn{3}{|c|}{$95.38 \%$} & & $83.23 \%$ \\
\hline & 1 & 6.88 & 14.75 & 10.8 & 5.88 & 9.41 & 7.83 & 4.25 & 6.46 & 4.96 \\
\hline & 2 & 11.75 & 20.24 & 17.4 & 9 & 14.06 & 11.95 & 7.64 & 12.29 & 9.94 \\
\hline & 3 & 15.46 & 24.58 & 21.71 & 11.13 & 17.28 & 14.66 & 10.43 & 15.07 & 13.04 \\
\hline & 4 & 18.34 & 25.54 & 23.81 & 12.83 & 18.45 & 15.8 & 12.77 & 17.43 & 15.86 \\
\hline & 5 & 20.57 & 25.74 & 24.25 & 14.3 & 19.7 & 17.17 & 14.77 & 18.65 & 17.95 \\
\hline & 6 & 22.32 & 26.53 & 24.81 & 15.64 & 22.73 & 19.54 & 16.46 & 19.89 & 20.18 \\
\hline & 7 & 23.75 & 27.37 & 25.53 & 16.88 & 24.6 & 20.75 & 17.88 & 21.05 & 21.8 \\
\hline & 8 & 25 & 28.08 & 26.24 & 18 & 26.48 & 21.72 & 19 & 21.31 & 21.47 \\
\hline & \multicolumn{3}{|c|}{ Representatividad } & \multicolumn{2}{|l|}{$92.15 \%$} & \multicolumn{3}{|c|}{$75.42 \%$} & & $88.82 \%$ \\
\hline \multirow{9}{*}{ 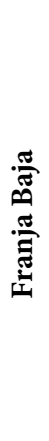 } & 1 & 6.13 & 10.13 & 7.75 & 5.5 & 10.89 & 7.5 & 3.13 & 3.85 & 3.27 \\
\hline & 2 & 10 & 17.13 & 13.98 & 9.57 & 14.94 & 13.95 & 5.18 & 6.53 & 5.65 \\
\hline & 3 & 13.05 & 21.11 & 17.87 & 12.77 & 18.28 & 18.42 & 6.64 & 8.17 & 7.33 \\
\hline & 4 & 15.69 & 25.86 & 22.48 & 15.4 & 20.97 & 21.7 & 7.77 & 9.4 & 8.73 \\
\hline & 5 & 18.02 & 30.79 & 26.56 & 17.64 & 23.69 & 25.12 & 8.7 & 10.44 & 10.02 \\
\hline & 6 & 20.14 & 32.94 & 28.31 & 19.61 & 26.36 & 28.39 & 9.5 & 11.45 & 11.45 \\
\hline & 7 & 22.13 & 35.94 & 30.75 & 21.38 & 28.48 & 30.67 & 10.25 & 12.46 & 12.89 \\
\hline & 8 & 24 & 38.38 & 32.95 & 23 & 30.47 & 32.26 & 11 & 13.48 & 13.97 \\
\hline & \multicolumn{3}{|c|}{ Representatividad } & $67.68 \%$ & & & $73.39 \%$ & & & $80.17 \%$ \\
\hline
\end{tabular}




\section{Revista de CIENCIAS AMBIENTALES

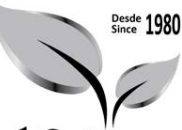 Tropical Journal of Environmental Sciences}
Revista de Ciencias Ambientales (Trop J Environ Sci) e-ISSN: 2215-3896
(Julio-Diciembre, 2021) . Vol 55(2): 197-228
DOI: https://doi.org/10.15359/rca.55-2.10
Open Access: www.revistas.una.ac.cr/ambientales e-mail: revista.ambientales@una.ac.cr Zuluaga-Carrero J. y Renjifo L.

Apéndice 2. Especies de árboles de cada tipo de vegetación en el enclave seco del cañón del Chicamocha, Colombia. BRA: bosque ripario alto, BRM: bosque ripario medio, BRB: bosque ripario bajo, BLA: bosque de ladera alto, BLM: bosque de ladera medio, BLB: bosque de ladera bajo, MA: matorral alto, MM: Matorral medio y MB: matorral bajo. Clasificación según APG III 2009.

Appendix 2. Tree species of each type of vegetation in the dry enclave of Chicamocha river canyon, Colombia. BRA: high riparian forest, BRM: medium riparian forest, BRB: low riparian forest, BLA: high hill forest, BLM: medium hill forest, BLB: low hill forest, MA: high scrub, MM: medium scrub and MB: low scrub. Classification according to APG III 2009.

\begin{tabular}{|c|c|c|c|c|c|c|c|c|c|c|}
\hline ORDEN / Familia & Especie & BRB & BLB & MB & BRM & BLM & MM & BRA & BLA & MA \\
\hline \multirow[t]{2}{*}{ Piperaceae } & Piper aducum & & & & & & & & 1 & \\
\hline & Piper lanceifolum & & & & & & & 9 & & \\
\hline Hernandiaceae & Gyrocarpus americanus & & & & 1 & & & & & \\
\hline \multirow[t]{2}{*}{ Annonaceae } & Annona cherimola & 8 & & & & & & & & \\
\hline & Annona squamosa & & & & & & 4 & & & \\
\hline Araceae & Xanthosoma sagittifolium & & & & & & & 2 & & \\
\hline Arecaceae & Acrocomia aculeata & & & & & & & 1 & & \\
\hline \multirow[t]{3}{*}{ Clusiaceae } & Clusia minor & & & & & & & 4 & 6 & 7 \\
\hline & Clusia rosea & & & & 1 & & & 5 & 18 & \\
\hline & Vismia baccifera & & & & & & & & 1 & \\
\hline Erythroxylaceae & Erythroxylum oxycarpum & & 1 & & & 9 & & & & \\
\hline \multirow[t]{5}{*}{ Euphorbiaceae } & Acalypha macrostachya & & & & & 1 & & & & \\
\hline & Croton caracasanus & & & & & & & & 2 & \\
\hline & Croton ferrugineus & & & & & 1 & 1 & & & 2 \\
\hline & Croton gossypiifolius & & & & & & & 2 & 1 & \\
\hline & Hura crepitans & & & & 4 & & & & & \\
\hline Hypericaceae & Vismia baccifera & & & & & & & 11 & 11 & 3 \\
\hline Lacistemataceae & Lacistema aggregatum & & 1 & & & & & & & \\
\hline \multirow[t]{2}{*}{ Malpighiaceae } & Byrsonima crassifolia & & & & & & & & & 14 \\
\hline & Malpighia glabra & & & & 3 & 1 & & & & \\
\hline Salicaceae & Casearia tremula & & 3 & & & & & & & \\
\hline
\end{tabular}

\begin{tabular}{|c|c|c|c|c|c|}
\hline 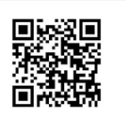 & (c) (i) & $\overbrace{\text { AMBENTIIIS }}$ & $\frac{O O \%}{20 \%}$ & $\frac{\text { UNA }}{\frac{\text { NNAESADAD }}{\text { UNIVESDID }}}$ & 219 \\
\hline
\end{tabular}




\section{Revista de

(Julio-Diciembre, 2021) . Vol 55(2): 197-228

DOI: https://doi.org/10.15359/rca.55-2.10

Open Access: www.revistas.una.ac.cr/ambientales e-mail: revista.ambientales@una.ac.cr Zuluaga-Carrero J. y Renjifo L.

\begin{tabular}{|c|c|c|c|c|c|c|c|c|c|c|}
\hline ORDEN / Familia & Especie & BRB & BLB & MB & BRM & BLM & MM & BRA & BLA & MA \\
\hline \multirow[t]{20}{*}{ Fabaceae } & Acacia farnesiana & 33 & 5 & 29 & & & 2 & & & \\
\hline & Albizia carbonaria & 1 & & & & & & & & \\
\hline & Albizia guachapele & 1 & & & 1 & & & & & \\
\hline & Albizia saman & & & & 1 & & & & & \\
\hline & Calliandra purdiaei & & & & & & & 48 & 3 & \\
\hline & Enterolobium schomburgkii & 4 & & & & 2 & & & & \\
\hline & Gliricidia sepium & 4 & & & & & 1 & & & \\
\hline & Haematoxylum brasiletto & 3 & 2 & 1 & 2 & 38 & 5 & & & \\
\hline & Inga vera & & & & & & & 5 & & \\
\hline & Machaerium biovulatum & & & & 2 & 2 & 2 & & & \\
\hline & Mimosa pudica & & & & 2 & & & & & \\
\hline & Parkinsonia praecox & & 10 & 21 & 1 & & & & & \\
\hline & Piptademia sp. & & & 9 & & & & & & \\
\hline & Pithecellobium dulce & & 12 & & & & & & & \\
\hline & Platymiscium pinnatum & 43 & 3 & & 4 & 10 & 14 & & & \\
\hline & Platypodium elegans & & & & & & & & 2 & \\
\hline & Prosopis juliflora & 1 & 31 & & & & & & & \\
\hline & Pseudopiptademia sp. & & & & & 1 & & & & \\
\hline & Senna pallida & & & & & 7 & 5 & & & \\
\hline & Senna tora & & & & 3 & & & & & \\
\hline \multirow[t]{2}{*}{ Cannabaceae } & Celtis iguanaea & & & & 3 & & & & & \\
\hline & Trema micrantha & & & & & & & 1 & & \\
\hline \multirow[t]{5}{*}{ Moraceae } & Ficus dendrocida & & 1 & & 6 & 2 & & & & \\
\hline & Ficus dugandii & 2 & & & & & 1 & & & \\
\hline & Ficus insipida & 1 & & & 6 & & 3 & 15 & 5 & \\
\hline & Ficus pallida & & & & 24 & & & & & \\
\hline & Maclura tinctoria & 1 & & & & & & 5 & & \\
\hline Urticaceae & Cecropia peltata & & & & & & & 2 & 5 & 2 \\
\hline \multirow[t]{5}{*}{ Melastomataceae } & Henriettea sp. & & & & & & & 3 & & \\
\hline & Miconia albicans & & & & & & & 2 & & 3 \\
\hline & Miconia rufescens & & & & & & & & 3 & \\
\hline & Miconia serrulata & & & & & & & & 2 & 12 \\
\hline & Tibouchina ciliaris & & & & & & & 1 & & \\
\hline \multirow[t]{7}{*}{ Myrtaceae } & Calycolpus moritzianus & & & & & & & & 6 & 11 \\
\hline & Eugenia biflora & & 1 & & & 12 & 3 & & & \\
\hline & Eugenia punicifolia & & & & 2 & 1 & & & & \\
\hline & Myrcia sp. & & & & & & & & 5 & \\
\hline & Myrcia splendens & & & & 10 & & & & & \\
\hline & Psidium guineense & & & & & & 1 & & & 14 \\
\hline & Sizygium jambos & & & & & & & 6 & 3 & 1 \\
\hline \multirow[t]{2}{*}{ Capparaceae } & Quadrella odoratissima & 1 & & 1 & & & & & & \\
\hline & Morisonia americana & & & 1 & & & & & & \\
\hline
\end{tabular}

\begin{tabular}{|c|c|c|c|c|}
\hline 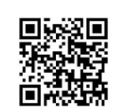 & (c) & $\Theta_{\text {AMEIENTIIIS }}$ & $\frac{O \%}{20}$ & 220 \\
\hline
\end{tabular}




\section{Revista de

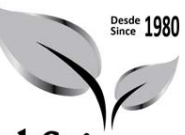 CIENCIAS AMBIENTALES Sciences}

Revista de Ciencias Ambientales (Trop J Environ Sci) e-ISSN: 2215-3896

(Julio-Diciembre, 2021) . Vol 55(2): 197-228

DOI: https://doi.org/10.15359/rca.55-2.10 Open Access: www.revistas.una.ac.cr/ambientales e-mail: revista.ambientales@una.ac.cr Zuluaga-Carrero J. y Renjifo L.

\begin{tabular}{|c|c|c|c|c|c|c|c|c|c|c|}
\hline ORDEN / Familia & Especie & BRB & BLB & MB & BRM & BLM & MM & BRA & BLA & MA \\
\hline \multirow[t]{5}{*}{ Malvaceae } & Ayenia magna & 1 & & & & & & & & \\
\hline & Cavanillesia chicamochae & & & & 8 & & & & 1 & \\
\hline & Ceiba pentandra & & & & 4 & & & & 3 & \\
\hline & Guazuma ulmifolia & 27 & 1 & 4 & & & & & & \\
\hline & Pseudobombax septenatum & & 4 & & & & & & & \\
\hline \multirow[t]{3}{*}{ Anacardiaceae } & Anacardium excelsum & 2 & & & 4 & & 4 & 6 & 4 & \\
\hline & Astronium graveolens & 13 & 7 & 11 & 27 & 27 & 14 & & & \\
\hline & Mangifera indica & & & & & & 2 & & & \\
\hline \multirow[t]{2}{*}{ Burseraceae } & Bursera graveolens & & 1 & & & 3 & & & & \\
\hline & Bursera simaruba & & 6 & & 6 & 11 & 4 & & & \\
\hline \multirow[t]{3}{*}{ Rutaceae } & Citrus limon & & & & & & 1 & & & \\
\hline & Zanthoxylum fagara & & 5 & & & & & & & \\
\hline & Zanthoxylum rhoifolium & 4 & & & 5 & & & 3 & 1 & \\
\hline \multirow[t]{2}{*}{ Sapindaceae } & Dodonaea viscosa & & & & & & 4 & & & 7 \\
\hline & Melicoccus bijugatus & & & 6 & & & & & & \\
\hline Santalaceae & Phoradendron quadrangulare & & & 7 & & & & & & \\
\hline Cactaceae & Stenocereus griseus & 1 & 1 & & & & & & & \\
\hline Polygonaceae & Ruprechtia ramiflora & 13 & 11 & 12 & & & & & & \\
\hline Primulaceae & Myrsine guianensis & & & & & 1 & & 13 & 19 & 21 \\
\hline \multirow[t]{2}{*}{ Apocynaceae } & Cascabela thevetia & 1 & & & & 1 & 5 & & & \\
\hline & Plumeria pudica & & & & 2 & & & & & \\
\hline \multirow[t]{2}{*}{ Rubiaceae } & Chicocca alba & & & & & & & & & 1 \\
\hline & Randia aculeata & 1 & 8 & & 2 & & & & & \\
\hline Bignoniaceae & Crescentia cujete & 2 & & & & & & & & \\
\hline Verbenaceae & Lippia origanoides & & 1 & & & 3 & 1 & & & 1 \\
\hline Solanaceae & Witheringia solanacea & & & & & & & & & 2 \\
\hline \multirow[t]{2}{*}{ Compositae } & Baccharis inamoena & & & & & & & & & 1 \\
\hline & Steiractinia sodiroi & & & & & & & & & 1 \\
\hline
\end{tabular}




\section{Revista de CIENCIAS AMBIENTALES Tropical Journal of Environmental Sciences}

Revista de Ciencias Ambientales (Trop J Environ Sci) e-ISSN: 2215-3896

(Julio-Diciembre, 2021) . Vol 55(2): 197-228

DOI: https://doi.org/10.15359/rca.55-2.10

Open Access: www.revistas.una.ac.cr/ambientales e-mail: revista.ambientales@una.ac.cr Zuluaga-Carrero J. y Renjifo L.

Apéndice 3. Estimadores no paramétricos de completitud de especies de aves en cada tipo de vegetación Cañón del río Chicamocha, Colombia. S(obs) hace referencia a la riqueza de especies observada durante el estudio en cada punto de conteo. Los estimadores ACE y Chaol representan la riqueza estimada, el porcentaje de representatividad se calculó con el promedio entre estos dos estimadores en cada punto de conteo.

Appendix 3. Non-parametric estimators of completeness of bird species in each type of vegetation dry enclave of Chicamocha river canyon, Colombia. S (obs) refers to the species richness observed during the study at each count point. The ACE and Chaol estimators represent the estimated richness, the percentage of representativeness was calculated with the average between these two estimators at each counting point.

\begin{tabular}{|c|c|c|c|c|c|c|c|c|c|c|}
\hline \multirow{10}{*}{ 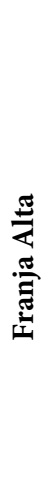 } & \multicolumn{4}{|c|}{ Bosque Ripario } & \multicolumn{3}{|c|}{ Bosque de Ladera } & \multicolumn{3}{|c|}{ Matorral } \\
\hline & Punto & S(obs) & ACE & Chao 1 & S(obs) & ACE & Chao 1 & S(obs) & ACE & Chao 1 \\
\hline & 1 & 14.5 & 19.92 & 16.6 & 14.88 & 20.8 & 17.5 & 17.63 & 25.88 & 21.56 \\
\hline & 2 & 22.54 & 26.22 & 24.3 & 25.04 & 32.61 & 29.52 & 28 & 34.14 & 31.1 \\
\hline & 3 & 27.96 & 31.8 & 29.98 & 32.66 & 40.71 & 37.13 & 35.32 & 40.25 & 37.69 \\
\hline & 4 & 31.99 & 35.59 & 33.81 & 38.66 & 46.3 & 43.73 & 40.99 & 44.9 & 42.49 \\
\hline & 5 & 35.14 & 38.73 & 36.93 & 43.46 & 51.04 & 48.79 & 45.55 & 49.5 & 46.87 \\
\hline & 6 & 37.75 & 41.58 & 39.88 & 47.36 & 55.05 & 53.07 & 49.29 & 52.69 & 50.43 \\
\hline & 7 & 40 & 44.14 & 42.16 & 50.5 & 57.39 & 55.11 & 52.38 & 54.93 & 53.02 \\
\hline & 8 & 42 & 45.98 & 43.9 & 53 & 58.7 & 56.65 & 55 & 56.81 & 55.27 \\
\hline \multirow{9}{*}{ 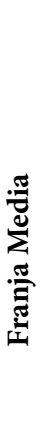 } & \multicolumn{3}{|c|}{ Representatividad } & \multicolumn{2}{|l|}{$93.50 \%$} & \multicolumn{3}{|c|}{$90.29 \%$} & & $98.16 \%$ \\
\hline & 1 & 11 & 16.14 & 12.96 & 11.25 & 16.98 & 13.37 & 15.5 & 19.47 & 17.07 \\
\hline & 2 & 18.04 & 22.67 & 20.23 & 19.07 & 24.41 & 21.23 & 24.96 & 27.33 & 25.72 \\
\hline & 3 & 22.98 & 27.67 & 25.21 & 24.88 & 30.23 & 27.43 & 31.95 & 35.2 & 32.74 \\
\hline & 4 & 26.89 & 32.05 & 29.18 & 29.37 & 34.45 & 31.73 & 37.53 & 41.47 & 38.46 \\
\hline & 5 & 30.27 & 36.54 & 32.99 & 32.98 & 37.52 & 35 & 42.14 & 45.92 & 43.1 \\
\hline & 6 & 33.36 & 39.83 & 35.75 & 36 & 40.24 & 37.85 & 46 & 50.14 & 47.37 \\
\hline & 7 & 36.25 & 43.36 & 38.56 & 38.63 & 43.1 & 40.64 & 49.25 & 52.97 & 50.44 \\
\hline & 8 & 39 & 46.85 & 41.1 & 41 & 45.66 & 43.32 & 52 & 54.95 & 52.75 \\
\hline \multirow{10}{*}{ 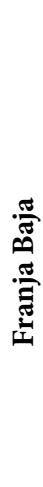 } & \multicolumn{3}{|c|}{ Representatividad } & $89.06 \%$ & & & $92.21 \%$ & & & $96.60 \%$ \\
\hline & 1 & 10 & 10.28 & 10.1 & 11.5 & 12.04 & 11.39 & 15.38 & 15.91 & 15.55 \\
\hline & 2 & 16.14 & 16.87 & 16.55 & 18.21 & 19.67 & 18.58 & 24.18 & 25.33 & 24.68 \\
\hline & 3 & 20.43 & 20.7 & 20.34 & 23.11 & 25.1 & 23.62 & 30.04 & 31.61 & 30.73 \\
\hline & 4 & 23.73 & 24.08 & 23.67 & 27.03 & 29.52 & 27.83 & 34.41 & 36.7 & 35.57 \\
\hline & 5 & 26.41 & 26.77 & 26.35 & 30.34 & 33.21 & 31.18 & 38.05 & 40.7 & 39.27 \\
\hline & 6 & 28.64 & 29.12 & 28.68 & 33.21 & 36.65 & 34.17 & 41.29 & 44.58 & 42.71 \\
\hline & 7 & 30.5 & 30.95 & 30.5 & 35.75 & 39.28 & 36.57 & 44.25 & 47.77 & 45.37 \\
\hline & 8 & 32 & 32.42 & 32 & 38 & 41.36 & 38.62 & 47 & 52.04 & 48.61 \\
\hline & \multicolumn{3}{|c|}{ Representatividad } & $99.32 \%$ & & & $95.13 \%$ & & & $93.54 \%$ \\
\hline
\end{tabular}

\begin{tabular}{|c|c|c|}
\hline 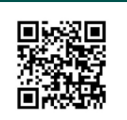 & (c) (i) $\$(?)$ & 222 \\
\hline
\end{tabular}




\section{Revista de CIENCIAS AMBIENTALES Tropical Journal of Environmental Sciences}

Revista de Ciencias Ambientales (Trop J Environ Sci) e-ISSN: 2215-3896

(Julio-Diciembre, 2021) . Vol 55(2): 197-228 DOI: https://doi.org/10.15359/rca.55-2.10

Open Access: www.revistas.una.ac.cr/ambientales e-mail: revista.ambientales@una.ac.cr Zuluaga-Carrero J. y Renjifo L.

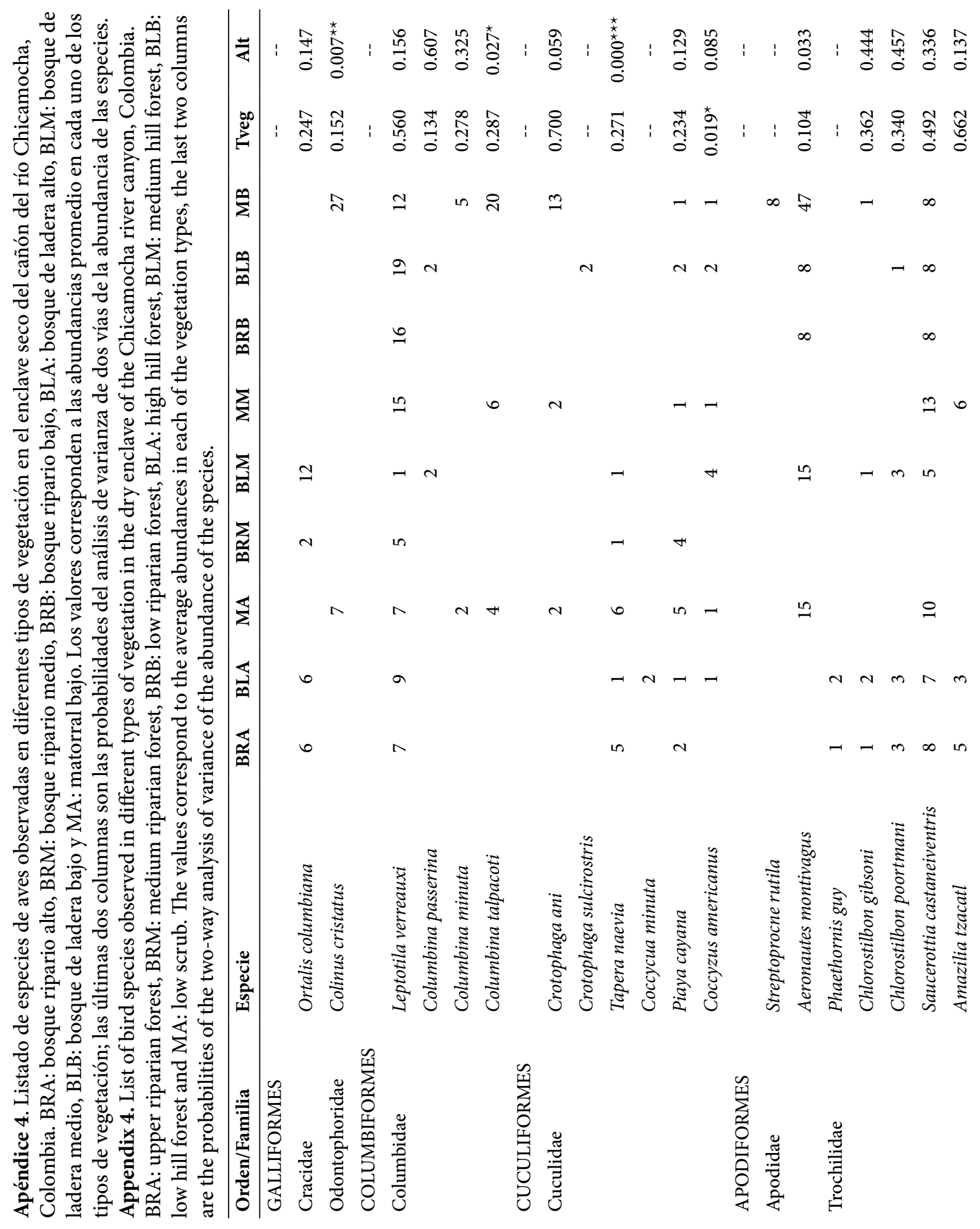

\begin{tabular}{|c|c|c|c|c|c|}
\hline 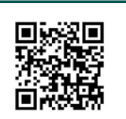 & (c) (1) & $\underset{\text { AMBEENAIIES }}{\longrightarrow}$ & $\frac{O \%}{\text { euna }}$ & 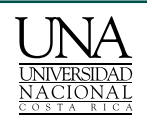 & 223 \\
\hline
\end{tabular}


Revista de
CIENCIAS AMBIENTALES
Tropical Journal of Environmental Sciences

Revista de Ciencias Ambientales (Trop J Environ Sci) e-ISSN: 2215-3896

(Julio-Diciembre, 2021) . Vol 55(2): 197-228 DOI: https://doi.org/10.15359/rca.55-2.10

Open Access: www.revistas.una.ac.cr/ambientales e-mail: revista.ambientales@una.ac.cr Zuluaga-Carrero J. y Renjifo L.

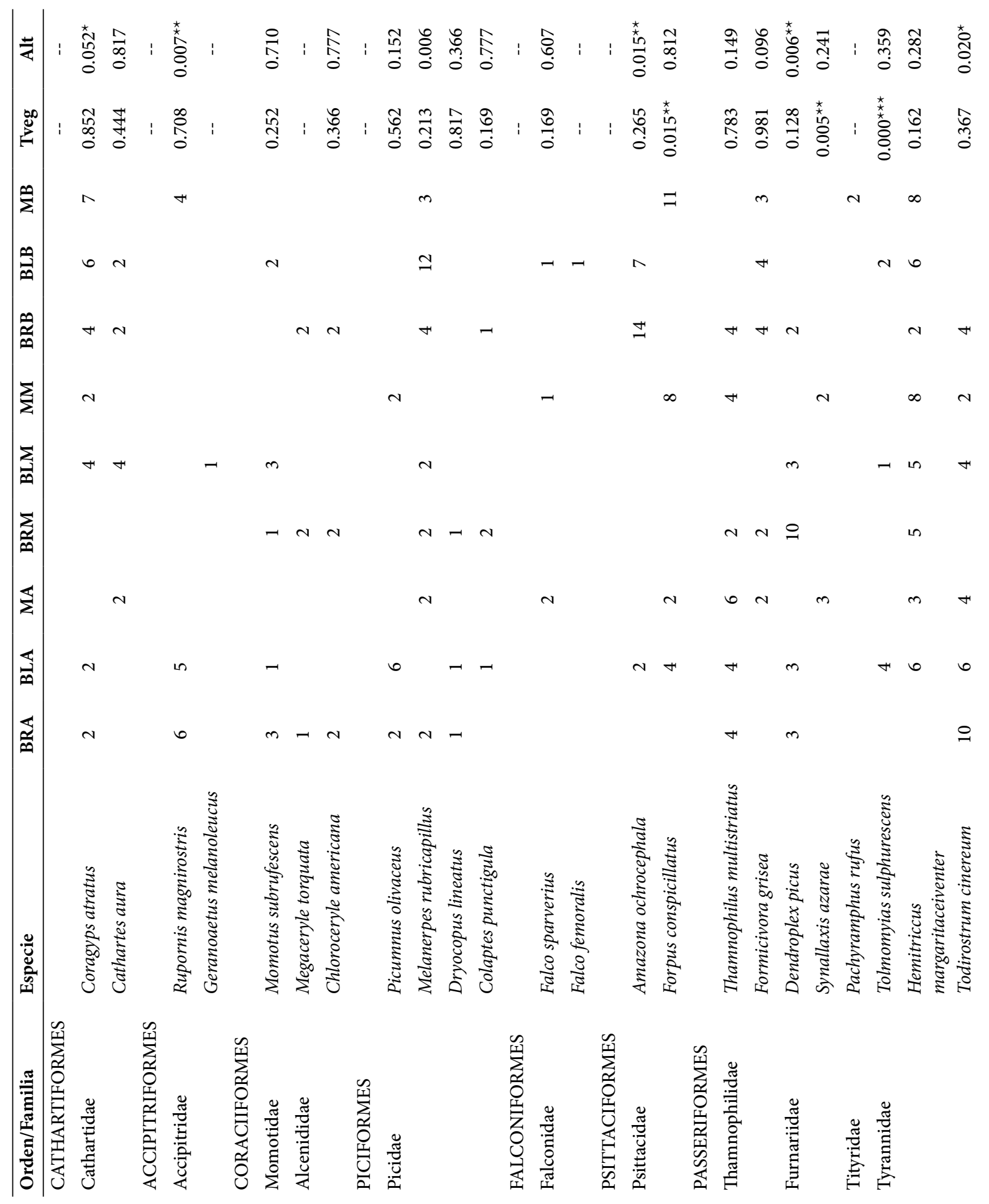

\begin{tabular}{|c|c|c|c|c|c|}
\hline 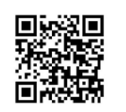 & (c) () & $\underset{\text { AMBENENALIES }}{\infty}$ & $\frac{1 \%}{2 \%}$ & $\frac{\text { UNA }}{\frac{\text { UNIEESIDAD }}{\frac{N A C I O N A L}{N A S T A R A L}}}$ & 224 \\
\hline
\end{tabular}




\section{Revista de CIENCIAS AMBIENTALES Tropical Journal of Environmental Sciences}

Revista de Ciencias Ambientales (Trop J Environ Sci) e-ISSN: 2215-3896

(Julio-Diciembre, 2021) . Vol 55(2): 197-228 DOI: https://doi.org/10.15359/rca.55-2.10

Open Access: www.revistas.una.ac.cr/ambientales e-mail: revista.ambientales@una.ac.cr Zuluaga-Carrero J. y Renjifo L.

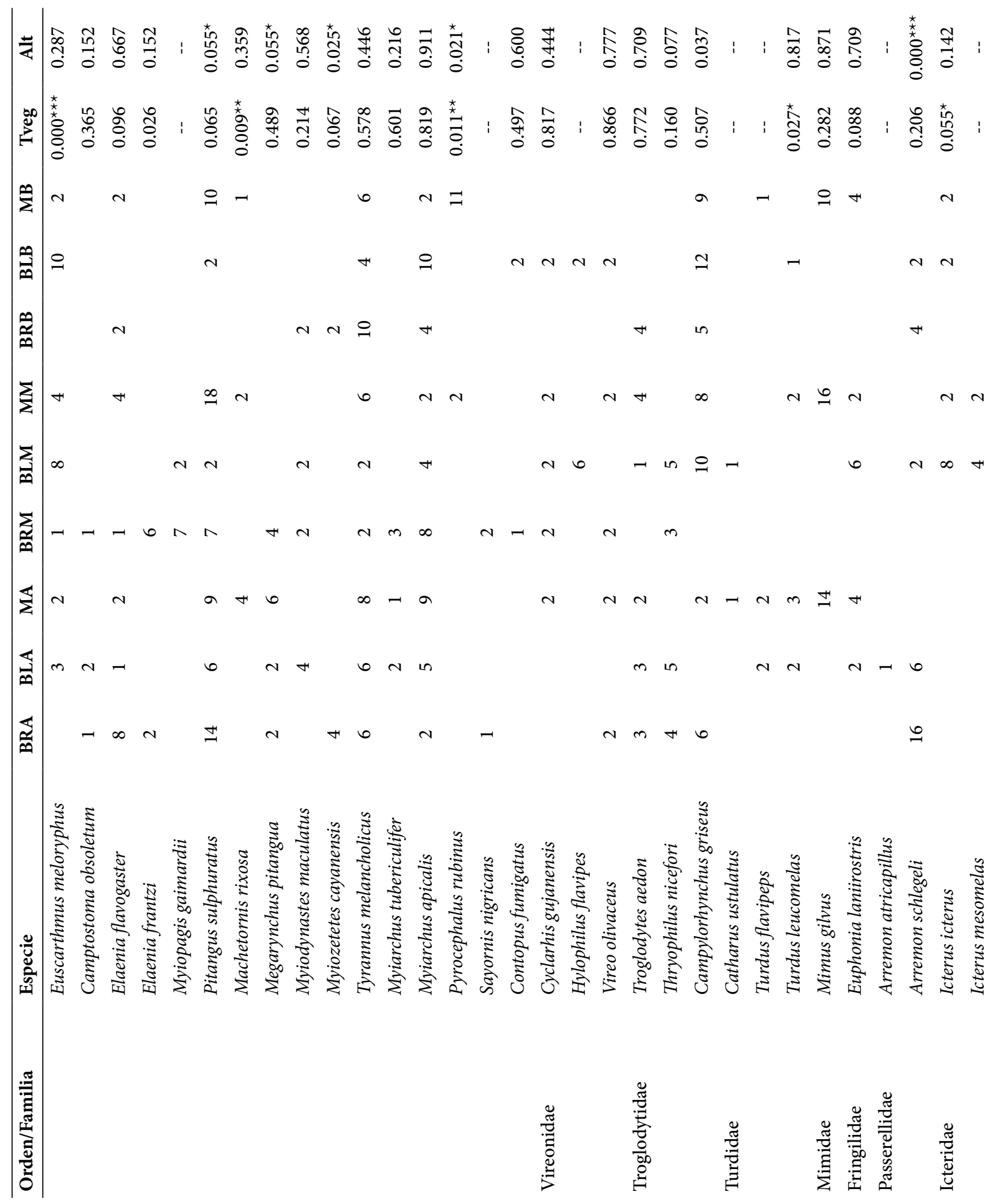

\begin{tabular}{|c|c|c|}
\hline 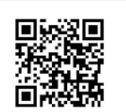 & (c) (1) & 225 \\
\hline
\end{tabular}




\section{Revista de CIENCIAS AMBIENTALES Tropical Journal of Environmental Sciences}

Revista de Ciencias Ambientales (Trop J Environ Sci) e-ISSN: 2215-3896

(Julio-Diciembre, 2021) . Vol 55(2): 197-228 DOI: https://doi.org/10.15359/rca.55-2.10 Open Access: www.revistas.una.ac.cr/ambientales e-mail: revista.ambientales@una.ac.cr Zuluaga-Carrero J. y Renjifo L.

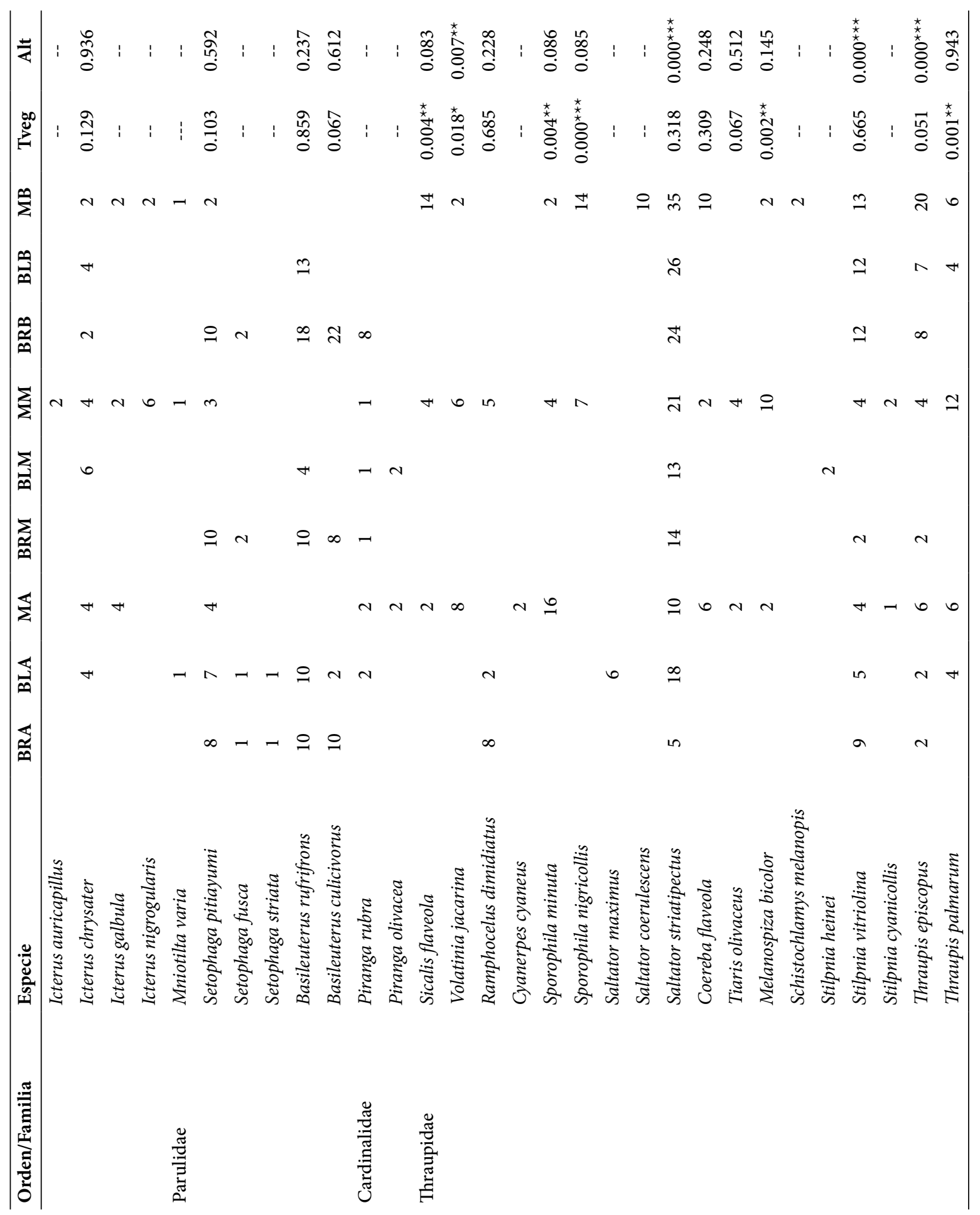

\begin{tabular}{|c|c|c|}
\hline 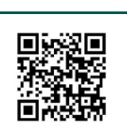 & (c) (i) () (2) & 226 \\
\hline
\end{tabular}




\section{Revista de CIENCIAS AMBIENTALES Tropical Journal of Environmental Sciences}

Revista de Ciencias Ambientales (Trop J Environ Sci) e-ISSN: 2215-3896

(Julio-Diciembre, 2021) . Vol 55(2): 197-228

DOI: https://doi.org/10.15359/rca.55-2.10

Open Access: www.revistas.una.ac.cr/ambientales e-mail: revista.ambientales@una.ac.cr Zuluaga-Carrero J. y Renjifo L.

Apéndice 5. Curvas rango abundancia de especies de aves presentes en nueve tipos de vegetación en el enclave seco del cañón del río Chicamocha. Las curvas se agruparon por fisionomía de vegetación para evaluar las diferencias en la distribución de sus abundancias. A. bosques riparios, B. bosques de ladera y C. matorrales en cada una de las cuales están representadas las franjas altitudinales. Los cuadros de la derecha corresponden con el color de cada curva y en ellos se encuentra el nombre de las seis especies más abundantes en cada tipo de vegetación; cada una de las curvas de rango abundancia de las especies de aves coincide con un modelo de abundancia. Las iniciales corresponden a cada tipo de vegetación, BRA: bosque ripario alto, BRM: bosque ripario medio, BRB: bosque ripario bajo, BLA: bosque de ladera alto, BLM: bosque de ladera medio, BLB: bosque de ladera bajo, MA: matorral alto, MM: Matorral medio y MB: matorral bajo.

Appendix 5. Range curves for abundance of bird species present in nine types of vegetation in the dry enclave of the Chicamocha river canyon. The curves were grouped by vegetation physiognomy to evaluate the differences in the distribution of their abundances. A. riparian forests, B. hillside forests and C. thickets in each of which the altitudinal bands are represented. The boxes on the right correspond to the color of each curve and they contain the name of the six most abundant species in each type of vegetation, each of the abundance range curves of the bird species coincides with an abundance model. The initials correspond to each type of vegetation, BRA: high riparian forest, BRM: medium riparian forest, BRB: low riparian forest, BLA: high slope forest, BLM: medium slope forest, BLB: low slope forest, MA: high scrub, MM: medium scrub and MB: low scrub.
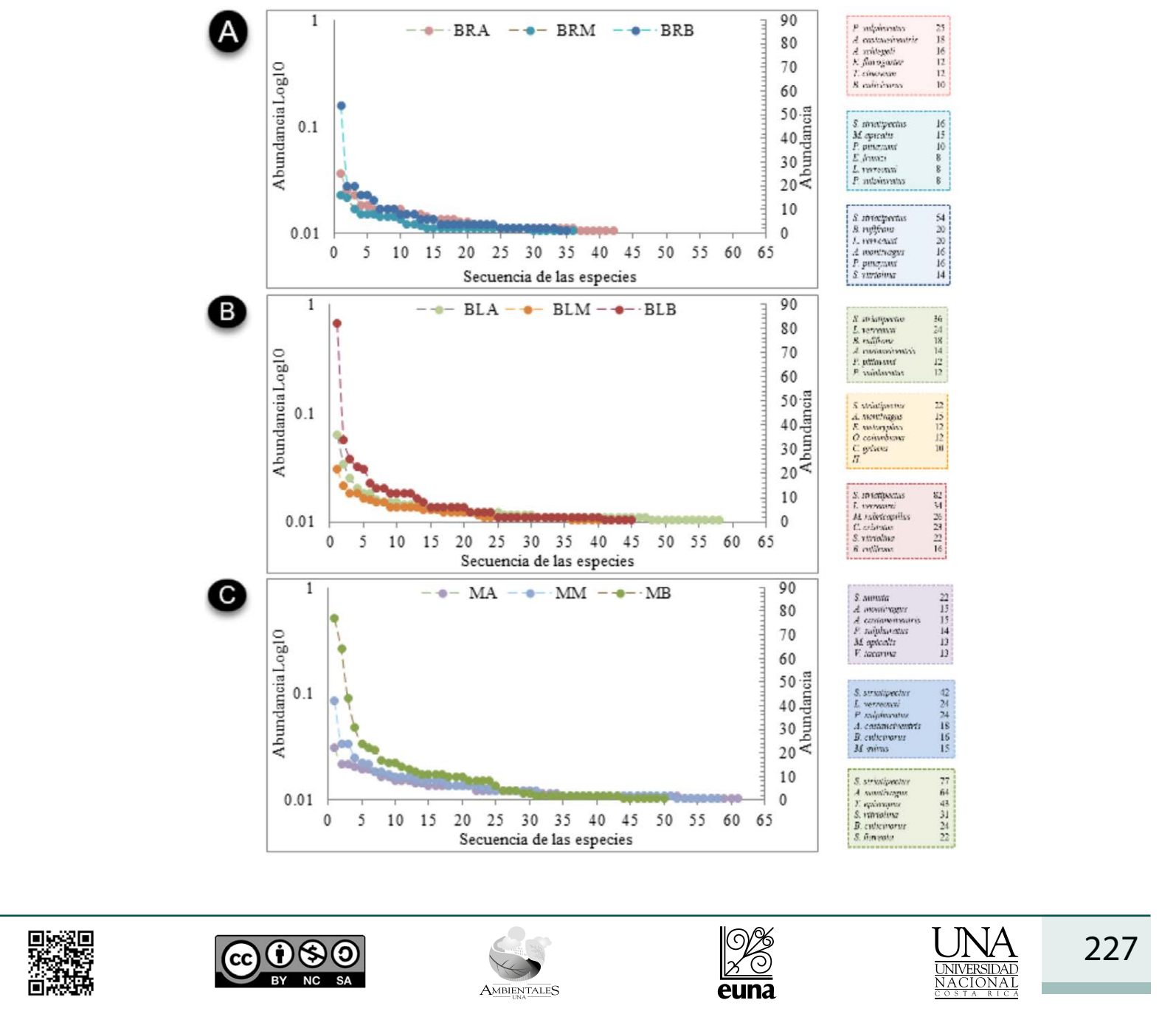


\section{Revista de CIENCIAS AMBIENTALES Tropical Journal of Environmental Sciences}

Revista de Ciencias Ambientales (Trop J Environ Sci) e-ISSN: 2215-3896

(Julio-Diciembre, 2021) . Vol 55(2): 197-228

DOI: https://doi.org/10.15359/rca.55-2.10

Open Access: www.revistas.una.ac.cr/ambientales e-mail: revista.ambientales@una.ac.cr Zuluaga-Carrero J. y Renjifo L.

Apéndice 6. Modelos de abundancia evaluados en cada uno de los tipos de vegetación presentes en el enclave seco del cañón del río Chicamocha, Colombia.

Appendix 6. Abundance models evaluated in each of the types of vegetation present in the dry enclave of the Chicamocha river canyon, Colombia.

\begin{tabular}{lccccccccc}
\hline & BRA & BRM & BRB & BLA & BLM & BLB & MA & MM & MB \\
\multicolumn{1}{c}{ Modelo } & Vara partida & Vara partida & Serie log & Vara partida & Serie log & Serie log & Vara partida & Serie log & Serie log \\
\hline Alpha & -- & -- & 10.7 & -- & 17.4 & 13.2 & -- & 19.5 & 13.5 \\
$\mathrm{X}$ & -- & -- & 0.961 & -- & 0.919 & 0.966 & -- & 0.948 & 0.975 \\
$\mathrm{Chi}^{\wedge} 2$ & 3.35 & 5.03 & 14.87 & 15.5 & 4.8 & 19.12 & 2.814 & 7.717 & 18.43 \\
$p$ & 1 & 0.99 & 0.82 & 0.99 & 1 & 0.89 & 1 & 1 & 0.97 \\
\hline
\end{tabular}

Apéndice 7. Índice de diversidad beta Williams en nueve tipos de vegetación presentes en el cañón del río Chicamocha, Colombia. Las iniciales corresponden a cada tipo de vegetación, BRA: bosque ripario alto, BRM: bosque ripario medio, BRB: bosque ripario bajo, BLA: bosque de ladera alto, BLM: bosque de ladera medio, BLB: bosque de ladera bajo, MA: matorral alto, MM: Matorral medio y MB: matorral bajo.

Appendix 7. Williams beta diversity index in nine types of vegetation present in the Chicamocha river canyon, Colombia. The initials correspond to each type of vegetation, BRA: high riparian forest, BRM: medium riparian forest, BRB: low riparian forest, BLA: high slope forest, BLM: medium slope forest, BLB: low slope forest, MA: high scrub, MM: medium scrub and MB: low scrub.

\begin{tabular}{cccccccccc}
\hline Tipos de vegetación & BRA & BRM & BRB & BLA & BLM & BLB & MA & MM & MB \\
\hline BRA & 0 & 0.22222 & 0.19231 & 0.13115 & 0.33333 & 0.33333 & 0.29487 & 0.2973 & 0.35616 \\
BRM & 0.22222 & 0 & 0.23529 & 0.17188 & 0.34921 & 0.35 & 0.23611 & 0.30667 & 0.34722 \\
BRB & 0.19231 & 0.23529 & 0 & 0.13115 & 0.24074 & 0.26923 & 0.1791 & 0.22388 & 0.25397 \\
BLA & 0.13115 & 0.17188 & 0.13115 & 0 & 0.22059 & 0.23188 & 0.32099 & 0.31169 & 0.31169 \\
BLM & 0.33333 & 0.34921 & 0.24074 & 0.22059 & 0 & 0.24074 & 0.25676 & 0.2973 & 0.33803 \\
BLB & 0.33333 & 0.35 & 0.26923 & 0.23188 & 0.24074 & 0 & 0.21429 & 0.23529 & 0.27692 \\
MA & 0.29487 & 0.23611 & 0.1791 & 0.32099 & 0.25676 & 0.21429 & 0 & 0.1791 & 0.1791 \\
MM & 0.2973 & 0.30667 & 0.22388 & 0.31169 & 0.2973 & 0.23529 & 0.1791 & 0 & 0.1875 \\
MB & 0.35616 & 0.34722 & 0.25397 & 0.31169 & 0.33803 & 0.27692 & 0.1791 & 0.1875 & 0 \\
\hline
\end{tabular}

